\title{
Optimal frame designs for multitasking devices with weight restrictions
}

\author{
María J. Benac, Pedro Massey, Mariano Ruiz and Demetrio Stojanoff * \\ Depto. de Matemática, FCE-UNLP, La Plata and IAM-CONICET, Argentina
}

\begin{abstract}
Let $\mathbf{d}=\left(d_{j}\right)_{j \in \mathbb{I}_{m}} \in \mathbb{N}^{m}$ be a finite sequence (of dimensions) and $\alpha=\left(\alpha_{i}\right)_{i \in \mathbb{I}_{n}}$ be a sequence of positive numbers (of weights), where $\mathbb{I}_{k}=\{1, \ldots, k\}$ for $k \in \mathbb{N}$. We introduce the ( $\alpha, \mathbf{d}$ )-designs i.e., $m$-tuples $\Phi=\left(\mathcal{F}_{j}\right)_{j \in \mathbb{I}_{m}}$ such that $\mathcal{F}_{j}=\left\{f_{i j}\right\}_{i \in \mathbb{I}_{n}}$ is a finite sequence in $\mathbb{C}^{d_{j}}, j \in \mathbb{I}_{m}$, and such that the sequence of non-negative numbers $\left(\left\|f_{i j}\right\|^{2}\right)_{j \in \mathbb{I}_{m}}$ forms a partition of $\alpha_{i}, i \in \mathbb{I}_{n}$. We characterize the existence of $(\alpha, \mathbf{d})$-designs with prescribed properties in terms of majorization relations. We show, by means of a finite-step algorithm, that there exist $(\alpha, \mathbf{d})$-designs $\Phi^{\mathrm{op}}=$ $\left(\mathcal{F}_{j}^{\mathrm{op}}\right)_{j \in \mathbb{I}_{m}}$ that are universally optimal; that is, for every convex function $\varphi:[0, \infty) \rightarrow[0, \infty)$ then $\Phi^{\text {op }}$ minimizes the joint convex potential induced by $\varphi$ among $(\alpha, \mathbf{d})$-designs, namely

$$
\sum_{j \in \mathbb{I}_{m}} \mathrm{P}_{\varphi}\left(\mathcal{F}_{j}^{\mathrm{op}}\right) \leq \sum_{j \in \mathbb{I}_{m}} \mathrm{P}_{\varphi}\left(\mathcal{F}_{j}\right)
$$

for every $(\alpha, \mathbf{d})$-design $\Phi=\left(\mathcal{F}_{j}\right)_{j \in \mathbb{I}_{m}}$, where $\mathrm{P}_{\varphi}(\mathcal{F})=\operatorname{tr}\left(\varphi\left(S_{\mathcal{F}}\right)\right)$; in particular, $\Phi^{\text {op }}$ minimizes both the joint frame potential and the joint mean square error among $(\alpha, \mathbf{d})$-designs. We show that in this case $\mathcal{F}_{j}^{\text {op }}$ is a frame for $\mathbb{C}^{d_{j}}$, for $j \in \mathbb{I}_{m}$. This corresponds to the existence of optimal encoding-decoding schemes for multitasking devices with energy restrictions.
\end{abstract}

AMS subject classification: 42C15, 15A60.

Keywords: frames, frame designs, convex potentials, majorization.

\section{Contents}

1 Introduction $\quad 2$

2 Preliminaries $4 . \ldots$

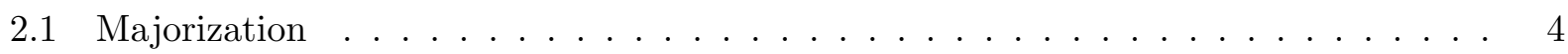

2.2 Frames and convex potentials $\ldots \ldots \ldots \ldots \ldots \ldots \ldots$

3 On $(\alpha, \mathbf{d})$-design problems $\quad 6$

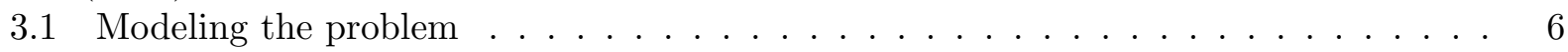

3.2 Main results . . . . . . . . . . . . . . . . . . . 7

4 Final comments and examples $\quad \mathbf{1 5}$

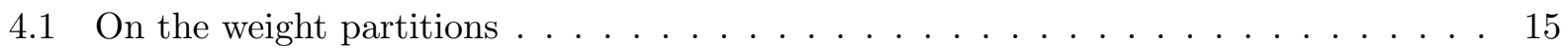

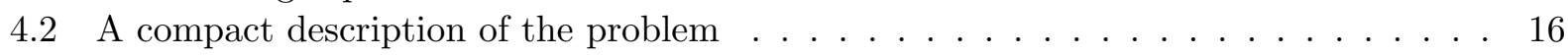

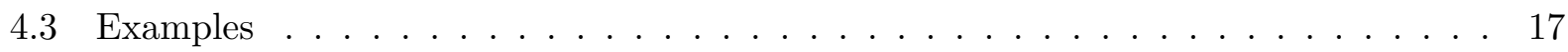

*Partially supported by CONICET (PICT ANPCyT 1505/15) and Universidad Nacional de La Plata (UNLP 11X829) e-mail addresses: mjbenac@gmail.com , massey@mate.unlp.edu.ar , mruiz@mate.unlp.edu.ar , demetrio@mate.unlp.edu.ar 


\section{Introduction}

A finite sequence $\mathcal{F}=\left\{f_{i}\right\}_{i \in \mathbb{I}_{n}}$ of vectors in $\mathbb{C}^{d}$ is a frame for $\mathbb{C}^{d}$ if $\mathcal{F}$ is a (possibly redundant) system of generators for $\mathbb{C}^{d}$. In this case, it is well known that there exist finite sequences $\mathcal{G}=\left\{g_{i}\right\}_{i \in \mathbb{I}_{n}}$ in $\mathbb{C}^{d}$ - the so called duals of $\mathcal{F}$ - such that

$$
f=\sum_{i \in \mathbb{I}_{n}}\left\langle f, f_{i}\right\rangle g_{i}=\sum_{i \in \mathbb{I}_{n}}\left\langle f, g_{i}\right\rangle f_{i} \quad \text { for } \quad f \in \mathbb{C}^{d} .
$$

Thus, we can encode/decode the vector $f$ in terms of the inner products $\left(\left\langle f, f_{i}\right\rangle\right)_{i \in \mathbb{I}_{n}} \in \mathbb{C}^{n}$ : (see $[7,12,13]$ and the references therein). The frame operator $S_{\mathcal{F}} \in \mathcal{M}_{d}(\mathbb{C})^{+}$is given by

$$
S_{\mathcal{F}} f=\sum_{i \in \mathbb{I}_{n}}\left\langle f, f_{i}\right\rangle f_{i} \quad \text { for } \quad f \in \mathbb{C}^{d} .
$$

If $S_{\mathcal{F}}$ is invertible (i.e. if $\mathcal{F}$ is a frame) the canonical dual of $\mathcal{F}$ is given by $g_{i}=S_{\mathcal{F}}^{-1} f_{i}$ for $i \in \mathbb{I}_{n}$; this dual plays a central role in applications since it has several optimal (minimal) properties within the set of duals of $\mathcal{F}$. Unfortunately, the computation of the canonical dual depends on finding $S_{\mathcal{F}}^{-1}$, which is a challenging task from the numerical point of view. A way out of this problem is to consider those frames $\mathcal{F}$ for which $S_{\mathcal{F}}^{-1}$ is easy to compute (e.g. tight frames). In general, the numerical stability of the computation of $S_{\mathcal{F}}^{-1}$ depends on the spread of the eigenvalues of $S_{\mathcal{F}}$. In [4] Benedetto and Fickus introduced a convex functional called the frame potential of a sequence $\mathcal{F}=\left\{f_{i}\right\}_{i \in \mathbb{I}_{n}}$ given by

$$
\mathrm{FP}(\mathcal{F})=\sum_{i, j \in \mathbb{I}_{n}}\left|\left\langle f_{i}, f_{j}\right\rangle\right|^{2} \geq 0
$$

In [4] the authors showed that under some normalization conditions, $\operatorname{FP}(\mathcal{F})$ provides an scalar measure of the spread of the eigenvalues of $\mathcal{F}$. More explicitly, the authors showed that the minimizers of $\mathrm{FP}$ among sequences $\mathcal{F}=\left\{f_{i}\right\}_{i \in \mathbb{I}_{n}}$ for which $\left\|f_{i}\right\|=1, i \in \mathbb{I}_{n}$, are exactly the $n / d$-tight frames. It is worth pointing out that these minimizers are also optimal for transmission through noisy channels (in which erasures of the frame coefficients may occur, see $[6,20]$ ).

In some applications of frame theory, we are drawn to consider frames $\mathcal{F}=\left\{f_{i}\right\}_{i \in \mathbb{I}_{n}}$ such that $\left\|f_{i}\right\|^{2}=\alpha_{i}, i \in \mathbb{I}_{n}$, for some prescribed sequence $\alpha=\left(\alpha_{i}\right)_{i \in \mathbb{I}_{n}} \in\left(\mathbb{R}_{>0}\right)^{n}$; this is known as the (classical) frame design problem. In practice, we can think of frames with prescribed norms as designs for encoding-decoding schemes to be applied by a device with some sort of energy restrictions (e.g. a device with limited access to energy power): in this case, control of the norms of the frame elements amounts to control the energy needed to apply the linear scheme.

It is then natural to wonder whether there are tight frames with norms prescribed by $\alpha$. This question has motivated the study of the frame design problem (see $[1,8,10,11,14,15,16,21]$ and $[17,18,23,22,25,26,27]$ for the more general frame completion problem with prescribed norms). It is well known that in some cases there are no tight frames in the class of sequences in $\mathbb{C}^{d}$ with norms prescribed by $\alpha$; in these cases, it is natural to consider minimizers of the frame potential within this class, since the eigenvalues of the frame operator of such minimizers have minimal spread (thus, inducing more stable linear reconstruction processes). These considerations lead to the study of optimal designs with prescribed structure. In [9], the authors compute the structure of such minimizers and show it resembles that of tight frames.

It is worth pointing out that there are other measures of the spread of the spectra of frame operators (e.g. the mean squared error (MSE)). It turns out that both the MSE and the FP lie within the class of convex potentials introduced in [24]. It is shown in [24] that there are solutions $\mathcal{F}^{\text {op }}$ to the frame design problem which are structural in the sense that they are minimizers of every convex potential (e.g. MSE and FP) among frames with squared norms prescribed by $\alpha$. A fundamental 
tool to show the existence of such structural optimal frame designs is the so-called majorization in $\mathbb{R}^{n}$, which is a partial order used in matrix analysis (see [5]).

Motivated originally in the study of optimal finitely generated shift invariant systems with norm restrictions, for a finitely generated shift invariant subspace of $L^{2}\left(\mathbb{R}^{d}\right)$ (see [2, Section 4.2.] and also [3]), in the present paper we consider extensions of the (classical) frame design problems as follows: given a finite sequence (of dimensions) $\mathbf{d}=\left(d_{j}\right)_{j \in \mathbb{I}_{m}} \in \mathbb{N}^{m}$ and a sequence (of weights) $\alpha=\left(\alpha_{i}\right)_{i \in \mathbb{I}_{n}} \in \mathbb{R}_{>0}^{n}$, we consider the set $\mathcal{D}(\alpha, \mathbf{d})$ of $(\alpha, \mathbf{d})$-designs. i.e. $m$-tuples $\Phi=\left(\mathcal{F}_{j}\right)_{j \in \mathbb{I}_{m}}$ such that each $\mathcal{F}_{j}=\left\{f_{i j}\right\}_{i \in \mathbb{I}_{n}}$ is a finite sequence in $\mathbb{C}^{d_{j}}$, and

$$
\sum_{j \in \mathbb{I}_{m}}\left\|f_{i j}\right\|^{2}=\alpha_{i} \quad \text { for } \quad i \in \mathbb{I}_{n}
$$

Notice that the restrictions on the norms above involve vectors in the (possibly different) spaces $f_{i j} \in \mathbb{C}^{d_{j}}$ for $j \in \mathbb{I}_{m}$. The $(\alpha, \mathbf{d})$-designs appear as the discretizations in the context of finitely generated shift invariant systems (see [2]). On the other hand, as in the case of frames with prescribed norms, $(\alpha, \mathbf{d})$-designs can be considered as encoding-decoding schemes to be applied by a multitasking device with some sort of energy restriction (e.g. due to isolation, or devices that are far from energy networks); in case $\mathcal{F}_{j}$ is a frame for $\mathbb{C}^{d_{j}}$ for $j \in \mathbb{I}_{m}$, then $\Phi=\left(\mathcal{F}_{j}\right)_{j \in \mathbb{I}_{m}}$ induces linear schemes in the spaces $\left(\mathbb{C}^{d_{j}}\right)_{j \in \mathbb{I}_{m}}$ that run in parallel. In this case, we want to control the overall energy needed (in each step of the encoding-decoding scheme) to apply simultaneously the $m$ linear schemes, through the restrictions in Eq.(4).

It is natural to consider those $(\alpha, \mathbf{d})$-designs that give rise to the more stable multitasking processes. In order the measure the overall stability of the family $\Phi=\left(\mathcal{F}_{j}\right)_{j \in \mathbb{I}_{m}}$ we can consider the joint frame potential of $\Phi$ or the joint MSE of $\Phi$ given by

$$
\operatorname{FP}(\Phi)=\sum_{j \in \mathbb{I}_{m}} \operatorname{FP}\left(\mathcal{F}_{j}\right) \quad, \quad \operatorname{MSE}(\Phi)=\sum_{j \in \mathbb{I}_{m}} \operatorname{MSE}\left(\mathcal{F}_{j}\right) \quad \text { respectively }
$$

More generally, given a convex function $\varphi:[0, \infty) \rightarrow[0, \infty)$ we introduce the joint convex potential $\mathrm{P}_{\varphi}(\Phi)$ induced by $\varphi$ (see Section 3.1 for details); this family of convex potentials (that contains the joint frame potential and joint MSE) provides natural measures of numerical stability of the family $\Phi=\left(\mathcal{F}_{j}\right)_{j \in \mathbb{I}_{m}}$. We remark that they are the same potentials considered in the previously mentioned context of finitely generated shift invariant systems in [2].

Given $(\alpha, \mathbf{d})$ as above, in this work we characterize the sequences of positive operators $S_{j} \in$ $\mathcal{M}_{d_{j}}(\mathbb{C})^{+}$for $j \in \mathbb{I}_{m}$, for which there exist $(\alpha, \mathbf{d})$-designs $\Phi=\left(\mathcal{F}_{j}\right)_{j \in \mathbb{I}_{m}}$ such that $S_{\mathcal{F}_{j}}=S_{j}$, for $j \in \mathbb{I}_{m}$. Our characterization is obtained in terms of the spectra of the operators $S_{j}$ and majorization relations, and it extends the well known solution of the classical frame design problem.

Then, we construct $(\alpha, \mathbf{d})$-designs $\Phi^{\mathrm{op}}$ that are optimal in $\mathcal{D}(\alpha, \mathbf{d})$; in this setting, optimality is measured in terms of joint convex potentials, as discussed above. The kernel of this problem is the computation of the optimal spectral structure among sequences in $\mathcal{D}(\alpha, \mathbf{d})$.

We point out that our approach to these problems is constructive; indeed, we describe a finite step algorithm that produces designs $\Phi \in \mathcal{D}(\alpha, \mathbf{d})$ with prescribed spectral structure and optimal designs $\Phi^{\mathrm{op}} \in \mathcal{D}(\alpha, \mathbf{d})$ as above. Moreover, we include several numerical examples of optimal $(\alpha, \mathbf{d})$-designs obtained with the implementation of our algorithm in MATLAB. We further obtain the uniqueness of the spectral structure of optimal $(\alpha, \mathbf{d})$-designs. Moreover, we show that the optimal spectral structure does not depend on the particular choice of the convex potential. As a consequence, our results generalize the results in [4, 9, 24].

The existence of optimal $(\alpha, \mathbf{d})$-designs as above settles in the affirmative a conjecture in $[2$, Section 4.2.] regarding the existence of optimal finitely generated shift invariant systems (for a finitely 
generated shift invariant subspace of $L^{2}\left(\mathbb{R}^{d}\right)$ ) with norm restrictions, with respect to convex potentials (see also [3]). On the other hand, our results have potential applications in comunication theory, e.g. in the study of the capacity of Multiple Input Multiple Output (MIMO) Additive White Gaussian Noise (AWGN) channels (see [19]).

The paper is organized as follows. In Section 2 we recall the notion of majorization together with some fundamental results about this pre-order. We also include some notions and results related with finite frame theory and convex potentials. In Section 3 we formalize the notion of $(\alpha, \mathbf{d})$ designs and describe in detail our main goals. In Section 3.2 we state and prove our main results, that include an effective characterization of the existence of $(\alpha, \mathbf{d})$-designs with prescribed spectral structure as well as the existence of (universal) optimal designs. The paper ends with Section 4, in which we present some general comments about the problems we studied, and several numerical examples that exhibit the properties of the optimal $(\alpha, \mathbf{d})$-designs computed with a finite step algorithm.

\section{Preliminaries}

In this section we introduce the notation, terminology and results from matrix analysis and frame theory that we will use throughout the paper. General references for these results are the texts [5] and $[7,12,13]$. In what follows we adopt the following

Notation and terminology. We let $\mathcal{M}_{k, d}(\mathcal{S})$ be the set of $k \times d$ matrices with coefficients in $\mathcal{S} \subset \mathbb{C}$ and write $\mathcal{M}_{d, d}(\mathbb{C})=\mathcal{M}_{d}(\mathbb{C})$ for the algebra of $d \times d$ complex matrices. We denote by $\mathcal{H}(d) \subset \mathcal{M}_{d}(\mathbb{C})$ the real subspace of selfadjoint matrices and by $\mathcal{M}_{d}(\mathbb{C})^{+} \subset \mathcal{H}(d)$ the cone of positive semidefinite matrices. We let $\mathcal{U}(d) \subset \mathcal{M}_{d}(\mathbb{C})$ denote the group of unitary matrices. For $d \in \mathbb{N}$, let $\mathbb{I}_{d}=\{1, \ldots, d\}$ and let $\mathbb{1}_{d}=(1)_{i \in \mathbb{I}_{d}} \in \mathbb{R}^{d}$ be the vector with all its entries equal to 1 .

Given $x=\left(x_{i}\right)_{i \in \mathbb{I}_{d}} \in \mathbb{R}^{d}$ we denote by $x^{\downarrow}=\left(x_{i}^{\downarrow}\right)_{i \in \mathbb{I}_{d}}$ (respectively $x^{\uparrow}=\left(x_{i}^{\uparrow}\right)_{i \in \mathbb{I}_{d}}$ ) the vector obtained by rearranging the entries of $x$ in non-increasing (respectively non-decreasing) order. We denote by $\left(\mathbb{R}^{d}\right)^{\downarrow}=\left\{x^{\downarrow}: x \in \mathbb{R}^{d}\right\},\left(\mathbb{R}_{\geq 0}^{d}\right)^{\downarrow}=\left\{x^{\downarrow}: x \in \mathbb{R}_{\geq 0}^{d}\right\}$ and analogously for $\left(\mathbb{R}^{d}\right)^{\uparrow}$ and $\left(\mathbb{R}_{\geq 0}^{d}\right)^{\uparrow}$.

Given a matrix $A \in \mathcal{H}(d)$ we denote by $\lambda(A)=\lambda^{\downarrow}(A)=\left(\lambda_{i}(A)\right)_{i \in \mathbb{I}_{d}} \in\left(\mathbb{R}^{d}\right)^{\downarrow}$ the eigenvalues of $A$ counting multiplicities and arranged in non-increasing order, and by $\lambda^{\uparrow}(A)$ the same vector but ordered in non-decreasing order. If $x, y \in \mathbb{C}^{d}$ we denote by $x \otimes y \in \mathcal{M}_{d}(\mathbb{C})$ the rank-one matrix given by $(x \otimes y) z=\langle z, y\rangle x$, for $z \in \mathbb{C}^{d}$.

\subsection{Majorization}

Next we recall the notion of majorization between vectors, that will play a central role throughout our work.

Definition 2.1. $\quad$ 1. Let $x, y \in \mathbb{R}^{d}$. We say that $x$ is submajorized by $y$, and write $x \prec_{w} y$, if

$$
\sum_{i \in \mathbb{I}_{j}} x_{i}^{\downarrow} \leq \sum_{i \in \mathbb{I}_{j}} y_{i}^{\downarrow} \quad \text { for every } \quad 1 \leq j \leq d .
$$

If $x \prec_{w} y$ and $\operatorname{tr} x=\sum_{i \in \mathbb{I}_{d}} x_{i}=\sum_{i \in \mathbb{I}_{d}} y_{i}=\operatorname{tr} y$, then $x$ is majorized by $y$, and write $x \prec y$.

2. Let $x \in \mathbb{R}_{\geq 0}^{n}$ and $y \in \mathbb{R}_{\geq 0}^{d}$ with $n>d$. Then we define the notions of $\prec$ and $\prec_{w}$ between the vectors $x$ and $y$ (of different size) by changing $y$ by $y \oplus 0_{n-d}:=(y, 0, \ldots, 0) \in \mathbb{R}^{n}$. Then

$$
x \prec y \quad \text { if } \quad \sum_{i \in \mathbb{I}_{n}} x_{i}=\sum_{i \in \mathbb{I}_{d}} y_{i} \quad \text { and } \quad \sum_{i \in \mathbb{I}_{j}} x_{i}^{\downarrow} \leq \sum_{i \in \mathbb{I}_{j}} y_{i}^{\downarrow} \quad \text { for } \quad 1 \leq j \leq d .
$$

and similarly one defines $y \prec x$. 
It is well known that majorization is related with the class $\mathcal{D S}(d)$ of doubly stochastic matrices i.e., formed by $D \in \mathcal{M}_{d}(\mathbb{C})$ with real non-negative entries such that each row sum and column sum equals one.

Theorem 2.2 (See $[5])$. Let $x, y \in \mathbb{R}^{d}$. Then

$$
x \prec y \quad \Longleftrightarrow \quad \text { there exists } \quad D \in \mathcal{D S}(d) \quad \text { such that } \quad x=D y .
$$

Remark 2.3. Let $x, y \in \mathbb{R}^{d}$ be such that $x \prec y$. Using [5, Theorem II.1.10] we get a finite step algorithm (based on the so-called T-transformations) that constructs $D \in \mathcal{D S}(d)$ such that $x=D y$.

Majorization is intimately related with tracial inequalities of convex functions. The following result summarizes these relations (see for example [5]):

Theorem 2.4. Let $x, y \in \mathbb{R}^{d}$. If $\varphi: I \rightarrow \mathbb{R}$ is a convex function defined on an interval $I \subseteq \mathbb{R}$ such that $x, y \in I^{d}$ then:

1. If $x \prec y$, then $\operatorname{tr} \varphi(x) \stackrel{\text { def }}{=} \sum_{i \in \mathbb{I}_{d}} \varphi\left(x_{i}\right) \leq \sum_{i \in \mathbb{I}_{d}} \varphi\left(y_{i}\right)=\operatorname{tr} \varphi(y)$.

2. If only $x \prec_{w} y$, but $\varphi$ is an increasing convex function, then still $\operatorname{tr} \varphi(x) \leq \operatorname{tr} \varphi(y)$.

3. If $x \prec y$ and $\varphi$ is a strictly convex function such that $\operatorname{tr} \varphi(x)=\operatorname{tr} \varphi(y)$ then, $x^{\downarrow}=y^{\downarrow}$.

\subsection{Frames and convex potentials}

In what follows we adopt the following

Notation and terminology: let $\mathcal{F}=\left\{f_{i}\right\}_{i \in \mathbb{I}_{n}}$ be a finite sequence in $\mathbb{C}^{d}$. Then,

1. $T_{\mathcal{F}} \in \mathcal{M}_{d, n}(\mathbb{C})$ is the synthesis operator given by $T_{\mathcal{F}} x=\sum_{i \in \mathbb{I}_{n}} x_{i} f_{i}$, for $x=\left(x_{i}\right)_{i \in \mathbb{I}_{n}} \in \mathbb{C}^{n}$.

2. $T_{\mathcal{F}}^{*} \in \mathcal{M}_{n, d}(\mathbb{C})$ is the analysis operator and it is given by $T_{\mathcal{F}}^{*} f=\left(\left\langle f, f_{i}\right\rangle\right)_{i \in \mathbb{I}_{n}}$, for $f \in \mathbb{C}^{d}$.

3. $S_{\mathcal{F}} \in \mathcal{M}_{d}(\mathbb{C})^{+}$denotes the frame operator of $\mathcal{F}$ and it is given by $S_{\mathcal{F}}=T_{\mathcal{F}} T_{\mathcal{F}}^{*}$. Hence,

$$
S_{\mathcal{F}} f=\sum_{i \in \mathbb{I}_{n}}\left\langle f, f_{i}\right\rangle f_{i}=\sum_{i \in \mathbb{I}_{n}}\left(f_{i} \otimes f_{i}\right) f \quad \text { for } \quad f \in \mathbb{C}^{d} .
$$

4. We say that $\mathcal{F}$ is a frame for $\mathbb{C}^{d}$ if it spans $\mathbb{C}^{d}$; equivalently, $\mathcal{F}$ is a frame for $\mathbb{C}^{d}$ if $S_{\mathcal{F}}$ is a positive invertible operator acting on $\mathbb{C}^{d}$.

In several applied situations it is desired to construct a finite sequence $\mathcal{G}=\left\{g_{i}\right\}_{i \in \mathbb{I}_{n}} \in\left(\mathbb{C}^{d}\right)^{n}$, in such a way that the spectra of the frame operator of $\mathcal{G}$ is given by some $\lambda \in\left(\mathbb{R}_{>0}^{d}\right)^{\downarrow}$ and the squared norms of the frame elements are prescribed by a sequence of positive numbers $\alpha=\left(\alpha_{i}\right)_{i \in \mathbb{I}_{n}}$. This is known as the (classical) frame design problem and it has been studied by several research groups (see for example $[1,8,10,11,14,15,16,21]$ ). The following result characterizes the existence of such frame designs in terms of majorization relations.

Theorem $2.5([1,23])$. Let $\lambda \in \mathbb{R}_{\geq 0}^{d}$ and consider $a=\left(a_{i}\right)_{i \in \mathbb{I}_{n}} \in\left(\mathbb{R}_{>0}^{n}\right)^{\downarrow}$. Then there exists a sequence $\mathcal{G}=\left\{g_{i}\right\}_{i \in \mathbb{I}_{n}}$ in $\mathbb{C}^{d}$ such that $\lambda\left(S_{\mathcal{G}}\right)=\lambda^{\downarrow}$ and $\left\|g_{i}\right\|^{2}=a_{i}$ for $i \in \mathbb{I}_{n}$ if and only if $a \prec \lambda$.

The previous result shows the flexibility of structured frame designs, which is important in applied situations. Also, numerical stability of the encoding-decoding scheme induced by a frame plays a role in applications; hence, a central problem in this area is to described the structured frame designs that maximize the stability of their encoding-decoding scheme. One of the most important 
(scalar) measures of stability is the so-called frame potential introduced by Benedetto and Fickus in [4] given by

$$
\operatorname{FP}(\mathcal{F})=\sum_{i, j \in \mathbb{I}_{n}}\left|\left\langle f_{i}, f_{j}\right\rangle\right|^{2}=\operatorname{tr}\left(S_{\mathcal{F}}^{2}\right) \quad \text { for } \quad \mathcal{F}=\left\{f_{i}\right\}_{i \in \mathbb{I}_{n}} \in\left(\mathbb{C}^{d}\right)^{n} .
$$

Benedetto and Fickus have shown that (under certain normalization conditions) minimizers of the frame potential induce the most stable encoding-decoding schemes. More generally, we can measure the stability of the scheme induced by the sequence $\mathcal{F}=\left\{f_{i}\right\}_{i \in \mathbb{I}_{n}} \in\left(\mathbb{C}^{d}\right)^{n}$ in terms of convex potentials. In order to introduce these potentials we consider the sets

$$
\operatorname{Conv}\left(\mathbb{R}_{\geq 0}\right)=\left\{\varphi: \mathbb{R}_{\geq 0} \rightarrow \mathbb{R}_{\geq 0}: \varphi \text { is a convex function }\right\}
$$

and $\operatorname{Conv}_{\mathbf{S}}\left(\mathbb{R}_{\geq 0}\right)=\left\{\varphi \in \operatorname{Conv}\left(\mathbb{R}_{\geq 0}\right): \varphi\right.$ is strictly convex $\}$.

Definition 2.6. Following [24] we consider the convex potential $\mathrm{P}_{\varphi}$ associated to $\varphi \in \operatorname{Conv}\left(\mathbb{R}_{\geq 0}\right)$, given by

$$
\mathrm{P}_{\varphi}(\mathcal{F})=\operatorname{tr} \varphi\left(S_{\mathcal{F}}\right)=\sum_{i \in \mathbb{I}_{d}} \varphi\left(\lambda_{i}\left(S_{\mathcal{F}}\right)\right) \quad \text { for } \quad \mathcal{F}=\left\{f_{i}\right\}_{i \in \mathbb{I}_{n}} \in\left(\mathbb{C}^{d}\right)^{n},
$$

where the matrix $\varphi\left(S_{\mathcal{F}}\right)$ is defined by means of the usual functional calculus.

Convex potentials allow us to model several well known measures of stability considered in frame theory. For example, in case $\varphi(x)=x^{2}$ for $x \in \mathbb{R}_{\geq 0}$ then $\mathrm{P}_{\varphi}$ is the Benedetto-Fickus frame potential; in case $\varphi(x)=x^{-1}$ for $x \in \mathbb{R}_{>0}$ then $\mathrm{P}_{\varphi}$ is known as the mean squared error (MSE).

Going back to the problem of stable designs, it is worth pointing out the existence of structured designs that are optimal with respect to every convex potential. Indeed, given $\alpha=\left(\alpha_{i}\right)_{i \in \mathbb{I}_{n}} \in \mathbb{R}_{\geq 0}^{n}$ and $d \in \mathbb{N}$ with $d \leq n$, the $\alpha$-torus is the set:

$$
\mathcal{B}_{\alpha, d}=\left\{\mathcal{F}=\left\{f_{i}\right\}_{i \in \mathbb{I}_{n}} \in\left(\mathbb{C}^{d}\right)^{n}:\left\|f_{i}\right\|^{2}=\alpha_{i}, i \in \mathbb{I}_{n}\right\} .
$$

We endow $\mathcal{B}_{\alpha, d}$ (which is a product space) with the product metric. The structure of (local) minimizers of convex potentials in $\mathcal{B}_{\alpha, d}$ has been extensively studied. The first results were obtained for the frame potential in [4] and in a more general context in [9]. The case of general convex potentials was studied in $[17,18,22,23,24,25,26,27]$ (in some cases in the more general setting of frame completion problems with prescribed norms).

\section{On $(\alpha, \mathbf{d})$-design problems}

We begin this section by introducing notation and terminology that allow us to model the $(\alpha, \mathbf{d})$ design problems, including the optimal design problem with prescribed weights. Then, we state and prove our main results for $(\alpha, \mathbf{d})$-designs.

\subsection{Modeling the problem}

Now we generalize the $\alpha$-torus to the multi-frames:

Definition 3.1. Let $\alpha=\left(\alpha_{i}\right)_{i \in \mathbb{I}_{n}} \in \mathbb{R}_{>0}^{n}$ and $\mathbf{d}=\left(d_{j}\right)_{j \in \mathbb{I}_{m}} \in\left(\mathbb{N}^{m}\right)^{\downarrow}$ be such that $d_{1} \leq n$.

1. An $(\alpha, \mathbf{d})$-design is an $m$-tuple

$$
\Phi=\left(\mathcal{F}_{j}\right)_{j \in \mathbb{I}_{m}}, \quad \text { where } \quad \mathcal{F}_{j}=\left\{f_{i j}\right\}_{i \in \mathbb{I}_{n}} \in\left(\mathbb{C}^{d_{j}}\right)^{n} \quad \text { for } \quad j \in \mathbb{I}_{m}
$$

and such that $\sum_{j \in \mathbb{I}_{m}}\left\|f_{i j}\right\|^{2}=\alpha_{i}$, for $i \in \mathbb{I}_{n}$. 
2. We denote by $\mathcal{D}(\alpha, \mathbf{d})$ the set of all $(\alpha, \mathbf{d})$-designs. We point out that (in order to simplify our description of the model) we consider $(\alpha, \mathbf{d})$-designs in a broad sense; namely, if $\Phi=$ $\left(\mathcal{F}_{j}\right)_{j \in \mathbb{I}_{m}} \in \mathcal{D}(\alpha, \mathbf{d})$ then $\mathcal{F}_{j}$ is not necessarily a frame for $\mathbb{C}^{d_{j}}$, for $j \in \mathbb{I}_{m}$.

3. In order to compare the overall stability of the linear encoding-decoding schemes induced by an $(\alpha, \mathbf{d})$-design we introduce the following potentials: Given $\varphi \in \operatorname{Conv}\left(\mathbb{R}_{\geq 0}\right)$ we consider the joint potential induced by $\varphi$ on $\Phi=\left(\mathcal{F}_{j}\right)_{j \in \mathbb{I}_{m}} \in \mathcal{D}(\alpha, \mathbf{d})$ given by

$$
\mathrm{P}_{\varphi}(\Phi)=\sum_{j \in \mathbb{I}_{m}} \mathrm{P}_{\varphi}\left(\mathcal{F}_{j}\right)=\sum_{j \in \mathbb{I}_{m}} \operatorname{tr} \varphi\left(S_{\mathcal{F}_{j}}\right)=\sum_{j \in \mathbb{I}_{m}} \sum_{i \in \mathbb{I}_{d_{j}}} \varphi\left(\lambda_{i}\left(S_{\mathcal{F}_{j}}\right)\right)
$$

Consider the notation and terminology of Definition 3.1. We can now describe the main problems that we consider in this work as follows:

P1. Determine necessary and sufficient conditions for the existence of $(\alpha, \mathbf{d})$-designs with prescribed spectral structure and describe algorithmic procedures to construct such designs, in case they exist.

P2. Given $\varphi \in \operatorname{Conv}\left(\mathbb{R}_{\geq 0}\right)$ determine the existence and structure of those $\Phi_{\varphi} \in \mathcal{D}(\alpha, \mathbf{d})$ that minimize the joint convex potential $\mathrm{P}_{\varphi}$ in $\mathcal{D}(\alpha, \mathbf{d})$, that is

$$
\mathrm{P}_{\varphi}\left(\Phi_{\varphi}\right)=\min \left\{\mathrm{P}_{\varphi}(\Phi): \Phi \in \mathcal{D}(\alpha, \mathbf{d})\right\}
$$

In this case we say that $\Phi_{\varphi}$ is an $\mathrm{P}_{\varphi^{-}}$-optimal $(\alpha, \mathbf{d})$-design. Determine whether these $\mathrm{P}_{\varphi^{-}}$ optimal $(\alpha, \mathbf{d})$-designs depend on the particular choice of $\mathrm{P}_{\varphi}$, for strictly convex functions $\varphi \in \operatorname{Conv}_{\mathrm{S}}\left(\mathbb{R}_{\geq 0}\right)$.

P3. Describe an algorithmic procedure that computes $\mathrm{P}_{\varphi}$-optimal $(\alpha, \mathbf{d})$-designs.

$\mathrm{P}$ 4. Characterize the $\mathrm{P}_{\varphi}$-optimal $(\alpha, \mathbf{d})$-designs in terms of some structural properties.

We will solve problems P1.-P4. In particular, we will show that if $\Phi_{\varphi}=\left(\mathcal{F}_{j}\right)_{j \in \mathbb{I}_{m}}$ is an $\mathrm{P}_{\varphi}$-optimal $(\alpha, \mathbf{d})$-design for $\varphi \in \operatorname{Conv}_{\mathbf{s}}\left(\mathbb{R}_{\geq 0}\right)$ then, $\mathcal{F}_{j}$ is a frame for $\mathbb{C}^{d_{j}}$ for each $j \in \mathbb{I}_{m}$ (see Section 3.2). Moreover, we will show that $\mathrm{P}_{\varphi}$-optimal $(\alpha, \mathbf{d})$-designs do not depend on the particular choice of $\mathrm{P}_{\varphi}$, for strictly convex functions $\varphi \in \mathrm{Conv}_{\mathrm{S}}\left(\mathbb{R}_{\geq 0}\right)$.

\subsection{Main results}

In this section we state and prove our main results; these include the existence of $(\alpha, \mathbf{d})$-designs with prescribed spectral structure, and designs with some special structure which turn out to be optimal designs in the sense of Problem (P2). We further show the uniqueness of the spectral structure of optimal $(\alpha, \mathbf{d})$-designs.

Our first main result characterizes the existence of $(\alpha, \mathbf{d})$-designs with prescribed spectral structure. We formalize problem $\mathrm{P} 1$. in terms of the following

Definition 3.2. Let $\mathbf{d}=\left(d_{j}\right)_{j \in \mathbb{I}_{m}} \in\left(\mathbb{N}^{m}\right)^{\downarrow}$ and $\alpha=\left(\alpha_{i}\right)_{i \in \mathbb{I}_{n}} \in\left(\mathbb{R}_{\geq 0}^{n}\right)^{\downarrow}$ be such that $d_{1} \leq n$. Let

$$
\mu_{j}=\left(\mu_{i, j}\right)_{i \in \mathbb{I}_{d_{j}}} \in\left(\mathbb{R}_{\geq 0}^{d_{j}}\right)^{\downarrow} \quad \text { for } \quad j \in \mathbb{I}_{m} \quad \text { and set } \quad \mathcal{M}:=\left\{\mu_{j}\right\}_{j \in \mathbb{I}_{m}} \in \prod_{j \in \mathbb{I}_{m}}\left(\mathbb{R}_{\geq 0}^{d_{j}}\right)^{\downarrow} .
$$

We say that the pair $(\alpha, \mathcal{M})$ is admissible if there exists $\Phi=\left(\mathcal{F}_{j}\right)_{j \in \mathbb{I}_{m}} \in \mathcal{D}(\alpha, \mathbf{d})$ such that

$$
\lambda\left(S_{\mathcal{F}_{j}}\right)=\mu_{j} \quad \text { for every } \quad j \in \mathbb{I}_{m} .
$$

In this case we denote $\mathcal{M}=\mathcal{M}_{\Phi}$. 
In order to obtain an effective characterization of admissibility, we introduce the notion of $(\alpha, m)$ weight partition matrix.

Remark 3.3. Let $\mathbf{d}=\left(d_{j}\right)_{j \in \mathbb{I}_{m}} \in\left(\mathbb{N}^{m}\right)^{\downarrow}$ and $\alpha=\left(\alpha_{i}\right)_{i \in \mathbb{I}_{n}} \in\left(\mathbb{R}_{\geq 0}^{n}\right)^{\downarrow}$ be such that $d_{1} \leq n$.

1. We consider the set of $(\alpha, m)$-weight partitions given by

$$
P_{\alpha, m}=\left\{A=\left(a_{i j}\right)_{i \in \mathbb{I}_{n}, j \in \mathbb{I}_{m}} \in \mathcal{M}_{n, m}(\mathbb{C}): a_{i j} \geq 0 \quad \text { and } \sum_{j \in \mathbb{I}_{m}} a_{i j}=\alpha_{i} \quad \text { for } \quad i \in \mathbb{I}_{n}\right\} .
$$

2. A sequence $\left(\mathcal{F}_{j}\right)_{j \in \mathbb{I}_{m}} \in \mathcal{D}(\alpha, \mathbf{d}) \Longleftrightarrow$ its matrix of weights

$$
A=\left\{\left\|f_{i j}\right\|^{2}\right\}_{i \in \mathbb{I}_{n}} \quad \mathbb{I}_{m} \in P_{\alpha, m} .
$$

Lemma 3.4 (A first characterization of admissible pairs). Consider a pair $(\alpha, \mathcal{M})$ as in Definition 3.2 above. Then the following conditions are equivalent.

1. The pair $(\alpha, \mathcal{M})$ is admissible.

2. There exists a matrix $A \in P_{\alpha, m}$ such that

$$
c_{j}(A) \prec \mu_{j} \quad \text { for every } \quad j \in \mathbb{I}_{m},
$$

where $c_{j}(A) \in \mathbb{R}_{\geq 0}^{n}$ denotes the $j$-th column of $A$.

In particular, the set of sequences $\mathcal{M}$ such that $(\alpha, \mathcal{M})$ is admissible is convex in $\prod_{j \in \mathbb{I}_{m}} \mathbb{R}^{d_{j}}$.

Proof. If the pair $(\alpha, \mathcal{M})$ is admissible, let $\left(\mathcal{F}_{j}\right)_{j \in \mathbb{I}_{m}} \in \mathcal{D}(\alpha, \mathbf{d})$ be such that $\lambda\left(S_{\mathcal{F}_{j}}\right)=\mu_{j}$, for $j \in \mathbb{I}_{m}$. Let $A \in P_{\alpha, m}$ be given by $c_{j}(A)=\left(\left\|f_{i j}\right\|^{2}\right)_{i \in \mathbb{I}_{n}}$, where $\mathcal{F}_{j}=\left\{f_{i j}\right\}_{i \in \mathbb{I}_{n}}$, for $j \in \mathbb{I}_{m}$. Then, by the Theorem $2.5, c_{j}(A) \prec \mu_{j}$ for $j \in \mathbb{I}_{m}$.

Conversely, assume that there exists $A \in P_{\alpha, m}$ with $c_{j}(A) \prec \mu_{j}$, for $j \in \mathbb{I}_{m}$. Then, again by Theorem 2.5, for each $j \in \mathbb{I}_{m}$ there exists $\mathcal{F}_{j}=\left\{f_{i j}\right\}_{i \in \mathbb{I}_{n}} \in\left(\mathbb{C}^{d_{j}}\right)^{n}$ such that $c_{j}(A)=\left(\left\|f_{i j}\right\|^{2}\right)_{i \in \mathbb{I}_{n}}$ and $\lambda\left(S_{\mathcal{F}_{j}}\right)=\mu_{j}$. Hence, $\left(\mathcal{F}_{j}\right)_{j \in \mathbb{I}_{m}} \in \mathcal{D}(\alpha, \mathbf{d})$ which shows that the pair $(\alpha, \mathcal{M})$ is admissible.

The following result provides an effective method to determine whether a given pair is admissible or not.

Theorem 3.5. Let $\mathbf{d}=\left(d_{j}\right)_{j \in \mathbb{I}_{m}} \in\left(\mathbb{N}^{m}\right)^{\downarrow}$ and $\alpha=\left(\alpha_{i}\right)_{i \in \mathbb{I}_{n}} \in\left(\mathbb{R}_{\geq 0}^{n}\right)^{\downarrow}$ be such that $d_{1} \leq n$. Given a sequence $\mathcal{M}=\left\{\mu_{j}\right\}_{j \in \mathbb{I}_{m}} \in \prod_{j \in \mathbb{I}_{m}}\left(\mathbb{R}_{\geq 0}^{d_{j}}\right)^{\downarrow}$, set

$$
\sigma_{\mathcal{M}}:=\sum_{j \in \mathbb{I}_{m}}\left(\mu_{j} \oplus 0_{d_{1}-d_{j}}\right) \in\left(\mathbb{R}_{\geq 0}^{d_{1}}\right)^{\downarrow}
$$

Then, the pair $(\alpha, \mathcal{M})$ is admissible if and only if $\alpha \prec \sigma_{\mathcal{M}}$.

Proof. Assume first that the pair $(\alpha, \mathcal{M})$ is admissible. Then, by Lemma 3.4, there exists $A \in P_{\alpha, m}$ such that $c_{j}(A)=\left(a_{i j}\right)_{i \in \mathbb{I}_{n}} \prec \mu_{j}=\left(\mu_{i, j}\right)_{i \in \mathbb{I}_{d_{j}}} \in\left(\mathbb{R}_{\geq 0}^{d_{j}}\right)^{\downarrow}$, for $j \in \mathbb{I}_{m}$. Fix an index $j \in \mathbb{I}_{m}$. Note that $n \geq d_{1}=\min \left\{n, d_{1}\right\}$. Hence, by hypothesis, for $k \in \mathbb{I}_{d_{1}}$ we have that

$$
\sum_{i=1}^{k} a_{i j} \leq \sum_{i=1}^{k}\left(c_{j}(A)^{\downarrow}\right)_{i} \leq \sum_{i=1}^{\min \left\{k, d_{j}\right\}} \mu_{i, j} \Longrightarrow \sum_{i=1}^{k} \alpha_{i}=\sum_{j \in \mathbb{I}_{m}} \sum_{i=1}^{k} a_{i j} \leq \sum_{j \in \mathbb{I}_{m}} \sum_{i=1}^{\min \left\{k, d_{j}\right\}} \mu_{i, j} .
$$


Notice that

$$
\sigma_{\mathcal{M}}=\left(\sigma_{i}\right)_{i \in \mathbb{I}_{d_{1}}}=\sum_{j \in \mathbb{I}_{m}}\left(\mu_{j} \oplus 0_{d_{1}-d_{j}}\right) \Longrightarrow \sum_{j \in \mathbb{I}_{m}} \sum_{i=1}^{\min \left\{k, d_{j}\right\}} \mu_{i, j}=\sum_{i=1}^{k} \sigma_{i}, \quad k \in \mathbb{I}_{d_{1}} .
$$

Then, Eq. (10) shows that $\alpha \prec \sigma_{\mathcal{M}}$ (the equality $\operatorname{tr} \sigma_{\mathcal{M}}=\operatorname{tr} \alpha$ is clear).

For the converse, in order to show that $(\alpha, \mathcal{M})$ is admissible, we prove that there exists $A \in P_{\alpha, m}$ such that $c_{j}(A) \prec \mu_{j}$, for $j \in \mathbb{I}_{m}$ (see Lemma 3.4). Indeed, since $\alpha \prec \sigma_{\mathcal{M}}$, by Theorem 2.2 there exists a doubly stochastic matrix $D \in \mathcal{D S}(n)$, such that $D\left(\sigma_{\mathcal{M}} \oplus 0_{n-d_{1}}\right)=\alpha$. Consider $A \in \mathcal{M}_{n, m}\left(\mathbb{R}_{\geq 0}\right)$ determined by $c_{j}(A)=D\left(\mu_{j} \oplus 0_{n-d_{j}}\right)$, for $j \in \mathbb{I}_{m}$. Notice that in this case by construction, $c_{j}(A) \prec \mu_{j} \oplus 0_{n-d_{j}} \Longrightarrow c_{j}(A) \prec \mu_{j}$ for $j \in \mathbb{I}_{m}$ and

$$
A \mathbb{1}_{m}=\sum_{j \in \mathbb{I}_{m}} c_{j}(A)=\sum_{j \in \mathbb{I}_{m}} D\left(\mu_{j} \oplus 0_{n-d_{j}}\right)=D\left(\sigma_{\mathcal{M}} \oplus 0_{n-d_{1}}\right)=\alpha .
$$

Thus, $A \in P_{\alpha, m}$ and the pair $(\alpha, \mathcal{M})$ is admissible.

Remark 3.6 (Finite-step algorithm for constructing $(\alpha, \mathbf{d})$-designs with prescribed spectral structure). With the notation of Theorem 3.5, assume that $\alpha \prec \sigma_{\mathcal{M}}$. Hence, in this case the pair $(\alpha, \mathcal{M})$ is admissible. By Remark 2.3, there is a finite step algorithm that constructs $D \in \mathcal{D S}(n)$ such that $\alpha=D\left(\sigma_{\mathcal{M}} \oplus 0_{n-d_{1}}\right)$. From the previous proof we see that if we consider $A \in \mathcal{M}_{n, m}\left(\mathbb{R}_{\geq 0}\right)$ determined by $c_{j}(A)=D\left(\mu_{j} \oplus 0_{n-d_{j}}\right)$, for $j \in \mathbb{I}_{m}$, then $A \in P_{\alpha, m}$. Moreover, by construction we have that $c_{j}(A) \prec \mu_{j} \oplus 0_{n-d_{j}}\left(\Longrightarrow c_{j}(A) \prec \mu_{j}\right)$ for $j \in \mathbb{I}_{m}$.

We can now apply finite step algorithms (such as the one-sided Bendel-Mickey algorithm, see $[10,11,14,16])$ and obtain $\mathcal{F}_{j}=\left\{f_{i j}\right\}_{i \in \mathbb{I}_{n}} \in\left(\mathbb{C}^{d_{j}}\right)^{n}$ such that $\left(\left\|f_{i j}\right\|^{2}\right)_{i \in \mathbb{I}_{n}}=c_{j}(A)$ and such that $\lambda\left(S_{\mathcal{F}_{j}}\right)=\mu_{j}$ for $j \in \mathbb{I}_{m}$. Therefore, we get $\Phi=\left(\mathcal{F}_{j}\right)_{j \in \mathbb{I}_{m}} \in \mathcal{D}\left(\alpha\right.$, d) such that $\mathcal{M}=\mathcal{M}_{\Phi}$ in a constructive way.

The following definition introduces an $m$-tuple of vectors (of eigenvalues), associated to every $(\alpha, \mathbf{d})$-design, and a large vector constructed from the juxtaposition of the elements of this set. These are going to be useful in proving the existence of optimal designs in terms of majorization relations, related with problem P2 above. (see Theorem 3.14 below).

Definition 3.7. Let $\mathbf{d}=\left(d_{j}\right)_{j \in \mathbb{I}_{m}} \in\left(\mathbb{N}^{m}\right)^{\downarrow}$ and $\alpha=\left(\alpha_{i}\right)_{i \in \mathbb{I}_{n}} \in\left(\mathbb{R}_{\geq 0}^{n}\right)^{\downarrow}$ be such that $d_{1} \leq n$. Let $\Phi=\left(\mathcal{F}_{j}\right)_{j \in \mathbb{I}_{m}} \in \mathcal{D}(\alpha, \mathbf{d})$ and let $S_{j}=S_{\mathcal{F}_{j}} \in \mathcal{M}_{d_{j}}(\mathbb{C})^{+}$denote the frame operators of $\mathcal{F}_{j}$, for $j \in \mathbb{I}_{m}$. We define

$$
\mathcal{M}_{\Phi}=\left\{\lambda\left(S_{j}\right)\right\}_{j \in \mathbb{I}_{m}} \in \prod_{j \in \mathbb{I}_{m}}\left(\mathbb{R}_{\geq 0}^{d_{j}}\right)^{\downarrow} \quad \text { and } \quad \Lambda_{\Phi}=\left(\lambda\left(S_{1}\right), \ldots, \lambda\left(S_{m}\right)\right) \in \mathbb{R}_{\geq 0}^{d},
$$

where $d=\operatorname{tr} \mathbf{d}=\sum_{j \in \mathbb{I}_{m}} d_{j}$ and each $\lambda\left(S_{j}\right) \in\left(\mathbb{R}_{\geq 0}^{d_{j}}\right)^{\downarrow}$ is the vector of eigenvalues of $S_{j}$, for $j \in \mathbb{I}_{m}$.

Recall that given a generic sequence $\mathcal{M} \in \prod_{j \in \mathbb{I}_{m}}\left(\mathbb{R}_{\geq 0}^{d_{j}}\right)^{\downarrow}$, we say that the pair $(\alpha, \mathcal{M})$ is admissible if there exists $\Phi \in \mathcal{D}(\alpha, \mathbf{d})$ such that $\mathcal{M}=\mathcal{M}_{\Phi}$, which in turns is equivalent to $\sigma_{\mathcal{M}} \prec \alpha$. We also remark that $\Lambda_{\Phi}$ is not an ordered vector. We shall use the specific order of its entries given in Eq.(11) in order to preserve the convexity properties given by Lemma 3.4.

Remark 3.8. Consider the notation in Definition 3.7. If $\varphi \in \operatorname{Conv}\left(\mathbb{R}_{\geq 0}\right)$ and $\mathrm{P}_{\varphi}$ denotes the joint convex potential induced by $\varphi$ (see Definition 2.6) then,

$$
\mathrm{P}_{\varphi}(\Phi)=\sum_{j \in \mathbb{I}_{m}} \mathrm{P}_{\varphi}\left(\mathcal{F}_{j}\right)=\sum_{j \in \mathbb{I}_{m}} \operatorname{tr}\left(\varphi\left(\lambda\left(S_{j}\right)\right)\right)=\sum_{\ell \in \mathbb{I}_{|d|}} \varphi\left(\left(\Lambda_{\Phi}\right)_{\ell}\right)=: \operatorname{tr}\left(\varphi\left(\Lambda_{\Phi}\right)\right) .
$$


Therefore, by Theorem 2.4 and Eq. (12), the existence of an (optimal) $(\alpha, \mathbf{d})$-design satisfying Eq. (7) for every $\varphi \in \operatorname{Conv}\left(\mathbb{R}_{\geq 0}\right)$ is equivalent to the existence of $\Psi=\left(\mathcal{G}_{j}\right)_{j \in \mathbb{I}_{m}} \in \mathcal{D}(\alpha, \mathbf{d})$ such that

$$
\Lambda_{\Psi} \prec \Lambda_{\Phi} \quad \text { for every } \quad \Phi=\left(\mathcal{F}_{j}\right)_{j \in \mathbb{I}_{m}} \in \mathcal{D}(\alpha, \mathbf{d}) .
$$

Remark 3.9. Consider the notation in Definition 3.7. In the rest of this section we shall show the existence of $(\alpha, \mathbf{d})$-designs $\Phi^{\mathrm{op}}=\left(\mathcal{F}_{j}^{\mathrm{op}}\right)_{j \in \mathbb{I}_{m}}$ that are optimal with respect to every joint convex potential (see Theorem 3.15). It turns out that these optimal designs have some special features.

In this remark we describe the special structure of the associated sequence $\mathcal{M}_{\Phi^{\text {op }}}$ (and introduce the necessary notation to describe this structure) in order to make more intelligible the next statements, which are intended to construct admissible pairs with this (optimal) structure. Let

$$
\mu_{j}^{\mathrm{op}}=\left(\mu_{i j}^{\mathrm{op}}\right)_{i \in \mathbb{I}_{d_{j}}}=\lambda\left(S_{\mathcal{F}_{j}^{\mathrm{op}}}\right) \in\left(\mathbb{R}_{\geq 0}^{d_{j}}\right)^{\downarrow} \quad \text { for every } \quad j \in \mathbb{I}_{m} .
$$

denote the eigenvalues of the frame operators of $\mathcal{F}_{j}^{\mathrm{op}}$. Then they must have the following structure: Each vector $\mu_{j}^{\text {op }} \in\left(\mathbb{R}_{\geq 0}^{d_{j}}\right)^{\downarrow}$ is a (truncated) copy of the first vector $\mu_{1}^{\text {op }} \in\left(\mathbb{R}_{\geq 0}^{d_{1}}\right)^{\downarrow}$, i.e.

$$
\mu_{i j}^{\text {op }}=\mu_{i 1}^{\text {op }} \quad \text { for every } \quad i \in \mathbb{I}_{j} \quad \text { and every } \quad j \in \mathbb{I}_{m} .
$$

In detail, let $\sigma\left(S_{\mathcal{F}_{1}^{\text {op }}}\right)=\left\{\gamma_{1}, \ldots, \gamma_{p}\right\}$, with $\gamma_{1}>\ldots>\gamma_{p} \geq 0$. Then there exist indexes $g_{0}=0<$ $g_{1}<\ldots<g_{p}=d_{1}$ (that we shall construct looking for admissibility) such that

$$
\left\{i \in \mathbb{I}_{d_{1}}: \mu_{i 1}=\gamma_{\ell}\right\}=\left\{i: g_{\ell-1}+1 \leq i \leq g_{\ell}\right\} \quad \text { for } \quad \ell \in \mathbb{I}_{p} .
$$

We define the following constants, which only depend on the data $\mathbf{d}=\left(d_{j}\right)_{j \in \mathbb{I}_{m}} \in\left(\mathbb{N}^{m}\right)^{\downarrow}$ :

$$
h_{i}:=\#\left\{j \in \mathbb{I}_{m}: d_{j} \geq i\right\} \quad \text { for } \quad i \in \mathbb{I}_{d_{1}} .
$$

Notice that,

$$
h:=\left(h_{i}\right)_{i \in \mathbb{I}_{d_{1}}}=\sum_{i=1}^{m} \mathbb{1}_{d_{i}} \oplus 0_{d_{1}-d_{i}} .
$$

\begin{tabular}{|c|c|c|c|c|}
\hline & $\begin{array}{c}\mu_{1}^{\mathrm{op}} \\
\downarrow\end{array}$ & $\begin{array}{c}\mu_{2}^{o p} \\
\downarrow\end{array}$ & $\begin{array}{c}\mu_{3}^{\mathrm{op}} \\
\downarrow\end{array}$ & $\begin{array}{c}\mu_{4}^{\text {op }} \\
\downarrow\end{array}$ \\
\hline$h_{1}=4$ & $\mu_{11}^{\mathrm{op}}$ & $\mu_{11}^{\mathrm{op}}$ & $\mu_{11}^{\text {op }}$ & $\mu_{11}^{\mathrm{op}}$ \\
\hline$h_{2}=4$ & $\mu_{21}^{o p}$ & $\mu_{21}^{\mathrm{op}}$ & $\mu_{21}^{\mathrm{op}}$ & $\mu_{21}^{\mathrm{op}}$ \\
\hline$h_{3}=3$ & $\mu_{31}^{\mathrm{op}}$ & $\mu_{31}^{\mathrm{op}}$ & $\mu_{31}^{\mathrm{op}}$ & $d_{4}=2$ \\
\hline$h_{4}=3$ & $\mu_{41}^{\mathrm{op}}$ & $\mu_{41}^{\mathrm{op}}$ & $\mu_{41}^{\text {op }}$ & \\
\hline$h_{5}=2$ & $\mu_{41}^{\mathrm{op}}$ & $\mu_{41}^{o \mathrm{op}}$ & $d_{3}=4$ & \\
\hline$h_{6}=1$ & $\mu_{41}^{\mathrm{op}}$ & $d_{2}=5$ & & \\
\hline
\end{tabular}

Using the relations in Eq. (13) we get that

$$
\Lambda_{\Phi \text { op }}^{\downarrow}=\left(\gamma_{\ell} \mathbb{1}_{r_{\ell}}\right)_{\ell \in \mathbb{I}_{p}} \quad \text { where } \quad r_{\ell}=\sum_{i=g_{\ell-1}+1}^{g_{\ell}} h_{i}, \quad \ell \in \mathbb{I}_{p} .
$$

We give an example of this situation for $m=4$ and $\mathbf{d}=(6,5,4,2)$ in Figure 1.

Figure 1: A graphic example of the structure of $\left(\mu_{j}^{\mathrm{op}}\right)_{j \in \mathbb{I}_{4}}$. 
For example, if we assume that

$$
\mu_{11}^{\mathrm{op}}=\mu_{21}^{\mathrm{op}}=\mu_{31}^{\mathrm{op}}=\gamma_{1}, \mu_{41}^{\mathrm{op}}=\mu_{51}^{\mathrm{op}}=\gamma_{2} \quad \text { and } \quad \mu_{61}^{\mathrm{op}}=\gamma_{3} \quad \text { with } \quad \gamma_{1}>\gamma_{2}>\gamma_{3}
$$

then we have: $g_{0}=0, g_{1}=3, g_{2}=5$ and hence, $r_{1}=11, r_{2}=5, r_{3}=1$; therefore, we compute $\Lambda_{\text {के }}^{\downarrow}=\left(\gamma_{1} \mathbb{1}_{11}, \gamma_{2} \mathbb{1}_{5}, \gamma_{3} \mathbb{1}_{1}\right) \in \mathbb{R}_{>0}^{17}$ in this case.

In order to obtain the our next main result, we consider the following

Notation 3.10. Let $\mathbf{d}=\left(d_{j}\right)_{j \in \mathbb{I}_{m}} \in\left(\mathbb{N}^{m}\right)^{\downarrow}$ and $\alpha=\left(\alpha_{i}\right)_{i \in \mathbb{I}_{n}} \in\left(\mathbb{R}_{>0}^{n}\right)^{\downarrow}$ be such that $d_{1} \leq n$. Let $h=\left(h_{i}\right)_{i \in \mathbb{I}_{d_{1}}}$ be defined as in Eq. (14). For $1 \leq s \leq t \leq d_{1}$, denote by $P_{s, t}$ and $Q_{t}$ the ratios

$$
P_{s, t}=\frac{\sum_{i=s}^{t} \alpha_{i}}{\sum_{i=s}^{t} h_{i}} \quad \text { and } \quad Q_{t}=\frac{\sum_{i=t}^{n} \alpha_{i}}{\sum_{i=t}^{d_{1}} h_{i}}
$$

The following result is a technical construction that we will use to build optimal $(\alpha, \mathbf{d})$-designs with a spectral picture as in Remark 3.9.

Theorem 3.11. Let $\mathbf{d}=\left(d_{j}\right)_{j \in \mathbb{I}_{m}} \in\left(\mathbb{N}^{m}\right)^{\downarrow}$ and $\alpha=\left(\alpha_{i}\right)_{i \in \mathbb{I}_{n}} \in\left(\mathbb{R}_{\geq 0}^{n}\right)^{\downarrow}$ be such that $d_{1} \leq n$. Consider the vector $h=\left(h_{i}\right)_{i \in \mathbb{I}_{d_{1}}}$ as in Eq. (15). Then, there exist

$$
p \in \mathbb{I}_{d_{1}} \quad \text { and } \quad g_{1}, \ldots, g_{p} \in \mathbb{N} \quad \text { with } \quad 0=g_{0}<g_{1}<\cdots<g_{p}=d_{1}
$$

such that, if we define $\gamma_{i}=P_{g_{i-1}+1, g_{i}}$, for $i \in \mathbb{I}_{p-1}$ and $\gamma_{p}=Q_{g_{p-1}+1}$ according with Eq. (17), then

1. $\gamma_{1}>\ldots>\gamma_{p}>0$;

2. They satisfy the following "block" majorizations:

$$
\begin{aligned}
&\left(\gamma_{i} h_{k}\right)_{k=g_{i-1}+1}^{g_{i}} \succ\left(\alpha_{i}\right)_{k=g_{i-1}+1}^{g_{i}} \quad \text { for } \quad i \in \mathbb{I}_{p-1} \quad \text { and } \\
&\left(\gamma_{p} h_{k}\right)_{k=g_{p-1}+1}^{d_{1}} \succ\left(\alpha_{i}\right)_{k=g_{p-1}+1}^{n} .
\end{aligned}
$$

In particular,

$$
\left(\gamma_{1} \mathbb{1}_{g_{1}-g_{0}}, \gamma_{2} \mathbb{1}_{g_{2}-g_{1}}, \ldots, \gamma_{p} \mathbb{1}_{g_{p}-g_{p-1}}\right) \circ h \succ \alpha,
$$

where $\circ$ denotes the entry-wise product.

Proof. First note that $d_{1}$ clearly satisfies $Q_{d_{1}} \geq P_{d_{1}, d_{1}}$. Then we can define the index

$$
s^{*}=\min \left\{j \in \mathbb{I}_{d_{1}}: Q_{j} \geq P_{j, k} \quad \text { for every } \quad j \leq k \leq d_{1}\right\}
$$

We denote $c=Q_{s^{*}}$. Therefore, by Eq. (17),

$$
c \sum_{i=s^{*}}^{k} h_{i} \geq \sum_{i=s^{*}}^{k} \alpha_{i} \quad \text { for every } \quad s^{*} \leq k \leq d_{1} .
$$

In other words, $\left(\alpha_{k}\right)_{k=s^{*}}^{n} \prec\left(h_{k} c\right)_{k=s^{*}}^{d_{1}}$. If $s^{*}=1$ then we set $p=1, g_{0}=0, g_{1}=d_{1}$ and $\gamma_{1}=c>0$. Then items 1. and 2. of the statement are satisfied in this case.

Otherwise, $s^{*}>1$ and we proceed to find the step $g_{1}$. First, we define $\gamma_{1}$ :

$$
\gamma_{1}=\max \left\{P_{1, k}: 1 \leq k \leq s^{*}-1\right\},
$$


and then we define $g_{1}$ as:

$$
g_{1}=\max \left\{j \in \mathbb{I}_{s^{*}-1}: P_{1, j}=\gamma_{1}\right\} .
$$

By construction, we obtain that

$$
\left(\gamma_{1} h_{i}\right)_{i=1}^{g_{1}} \succ\left(\alpha_{i}\right)_{i=1}^{g_{1}} .
$$

Now, if $g_{1}=s^{*}-1$ then we set $p=2, g_{0}=0, g_{2}=d_{1}$ and $\gamma_{2}=c>0$.

Otherwise, $g_{1}<s^{*}-1$ (and having the index $s^{*}$ fixed), we define $g_{2}$ in a similar way:

$$
\gamma_{2}=\max \left\{P_{g_{1}+1, k}: g_{1}<k \leq s^{*}-1\right\}
$$

and then,

$$
g_{2}=\max \left\{g_{1}+1 \leq j \leq s^{*}-1: P_{g_{1}+1, j}=\gamma_{2}\right\} .
$$

Again, by construction we have that

$$
\left(\gamma_{2} h_{i}\right)_{i=g_{1}+1}^{g_{2}} \succ\left(\alpha_{i}\right)_{i=g_{1}+1}^{g_{2}} .
$$

We claim that $\gamma_{1}>\gamma_{2}$. Indeed, suppose that $P_{g_{1}+1, g_{2}}=\gamma_{2} \geq \gamma_{1}=P_{1, g_{1}}$. Then,

$$
\begin{aligned}
P_{1, g_{2}}-P_{1, g_{1}}= & \frac{\sum_{i=1}^{g_{1}} \alpha_{i}+\sum_{i=g_{1}+1}^{g_{2}} \alpha_{i}}{\sum_{i=1}^{g_{1}} h_{i}+\sum_{i=g_{1}+1}^{g_{2}} h_{i}}-\frac{\sum_{i=1}^{g_{1}} \alpha_{i}}{\sum_{i=1}^{g_{1}} h_{i}}= \\
= & \frac{\left(\sum_{i=1}^{g_{1}} h_{i}\right)\left(\sum_{i=g_{1}+1}^{g_{2}} \alpha_{i}\right)-\left(\sum_{i=g_{1}+1}^{g_{2}} h_{i}\right)\left(\sum_{i=1}^{g_{1}} \alpha_{i}\right)}{\sum_{i=1}^{g_{1}} h_{i}\left(\sum_{i=1}^{g_{1}} h_{i}+\sum_{i=g_{1}+1}^{g_{2}} h_{i}\right)} \\
= & \frac{\left(\sum_{i=1}^{g_{1}} h_{i}\right)\left(\sum_{i=g_{1}+1}^{g_{2}} h_{i}\right)\left(\gamma_{2}-\gamma_{1}\right)}{\sum_{i=1}^{g_{1}} h_{i}\left(\sum_{i=1}^{g_{1}} h_{i}+\sum_{i=g_{1}+1}^{g_{2}} h_{i}\right)} \geq 0 .
\end{aligned}
$$

Hence, $P_{1, g_{2}}=P_{1, g_{1}}=\gamma_{1}$ which contradicts the definition of $g_{1}$, so the claim is proved.

We can continue inductively with this process, that is, once we find $g_{k-1}<s^{*}-1$ we compute first $\gamma_{k}$ as the maximum among $P_{g_{k-1}+1, l}$, with $g_{k-1}+1 \leq l \leq s^{*}-1$ and then define $g_{k} \leq s^{*}-1$ as the maximum index $g_{k-1}+1 \leq l \leq s^{*}-1$ such that $P_{g_{k-1}+1, l}=\gamma_{k}$. As before, this construction guarantees the corresponding block majorization.

Notice that in the last step, corresponding to the $p-1$ iteration of the process, we necessarily have $g_{p-1}=s^{*}-1$. Define $\gamma_{p}=Q_{g_{p-1}+1}=c>0$ and $g_{p}=d_{1}$.

By construction, and the previous remarks we have that $\gamma_{1}>\gamma_{2}>\ldots>\gamma_{p-1}$ and item 18 is satisfied. It remains to prove that $\gamma_{p-1}>\gamma_{p}$.

Suppose, on the contrary, that $\gamma_{p} \geq \gamma_{p-1}$. Consider $\bar{c}=Q_{g_{p-2}+1}$. Clearly, $\bar{c}$ is a convex combination of $\gamma_{p}$ and $\gamma_{p-1}$, say $\bar{c}=t \gamma_{p-1}+(1-t) \gamma_{p}$. In particular, $\gamma_{p-1} \leq \bar{c} \leq \gamma_{p}$. Therefore, we have that

$$
P_{g_{p-2}+1, l} \leq \gamma_{p-1} \leq \bar{c}, \text { for } g_{p-2}+1 \leq l \leq g_{p-1}
$$

Let $g_{p-1}+1 \leq l<d_{1}$, and denote by $A=\sum_{i=g_{p-2}+1}^{g_{p-1}} h_{i}, B=\sum_{i=g_{p-1}+1}^{l} h_{i}$ and $C=\sum_{i=l+1}^{d_{1}} h_{i}$.

Notice that, with this notation, in the convex combination that generates $\bar{c}$, we have $t=\frac{A}{A+B+C}$. Then, since $\gamma_{p-1} \leq \gamma_{p}$,

$$
\frac{A}{A+B} \gamma_{p-1}+\frac{B}{A+B} \gamma_{p} \leq \frac{A}{A+B+C} \gamma_{p-1}+\frac{B+C}{A+B+C} \gamma_{p}
$$


Hence, since by definition of $\gamma_{p}$ we have $P_{g_{p-1}+1, l} \leq \gamma_{p}$, we obtain

$$
\frac{A}{A+B} \gamma_{p-1}+\frac{B}{A+B} P_{g_{p-1}+1, l} \leq \frac{A}{A+B+C} \gamma_{p-1}+\frac{B+C}{A+B+C} \gamma_{p}
$$

since $\frac{A}{A+B} \gamma_{p-1}+\frac{B}{A+B} P_{g_{p-1}+1, l}=P_{g_{p-2}+1, l}$ and $\frac{A}{A+B+C} \gamma_{p-1}+\frac{B+C}{A+B+C} \gamma_{p}=\bar{c}$, we deduce

$$
P_{g_{p-2}+1, l} \leq \bar{c}, \quad \text { for } \quad g_{p-1}+1 \leq l \leq d_{1}
$$

Therefore, Eqs. (21), (23) imply that, for $j=g_{p-2}+1<s^{*}=g_{p-1}+1$,

$$
P_{j, l} \leq Q_{j}, \quad \text { for } \quad l=j, \ldots, d_{1}
$$

which contradicts the construction of $s^{*}$. So we can conclude that $\gamma_{p-1}>\gamma_{p}$ and the theorem is proved.

Next, we introduce the following vectors associated to a pair $(\alpha, \mathbf{d})$.

Definition 3.12. Let $\mathbf{d}=\left(d_{j}\right)_{j \in \mathbb{I}_{m}} \in\left(\mathbb{N}^{m}\right)^{\downarrow}$ and $\alpha=\left(\alpha_{i}\right)_{i \in \mathbb{I}_{n}} \in\left(\mathbb{R}_{>0}^{n}\right)^{\downarrow}$ be such that $d_{1} \leq n$. Let $0=g_{0}<\cdots<g_{p}=d_{1}$ and $\gamma_{1}>\cdots>\gamma_{p}>0$ be as in Theorem 3.11. Then, we set:

1. $\mu_{1}^{\mathrm{op}}=\left(\mu_{i 1}^{\mathrm{op}}\right)_{i \in \mathbb{I}_{d_{1}}}=\left(\gamma_{k} \mathbb{1}_{g_{k}-g_{k-1}}\right)_{k=1}^{p} \in\left(\mathbb{R}_{>0}^{d_{1}}\right)^{\downarrow}$;

2. For $j>1$ set $\mu_{j}^{\mathrm{op}}=\left(\mu_{i j}^{\mathrm{op}}\right)_{i \in \mathbb{I}_{d_{j}}} \in\left(\mathbb{R}_{>0}^{d_{j}}\right)^{\downarrow}$ such that $\mu_{i j}^{\mathrm{op}}=\mu_{i 1}^{\mathrm{op}}$ for $i \in \mathbb{I}_{d_{j}}$.

3. Denote $\mathcal{M}^{\mathrm{op}}=\left\{\mu_{j}^{\mathrm{op}}\right\}_{j \in \mathbb{I}_{m}} \in \prod_{j \in \mathbb{I}_{m}}\left(\mathbb{R}_{>0}^{d_{j}}\right)^{\downarrow}$.

At this point, the vector $\mu_{1}^{\text {op }}$ (and so the sequence $\mathcal{M}^{\mathrm{op}}$ ) depends on the choice of the indexes $0=g_{0}<\cdots<g_{p}=d_{1}$ from Theorem 3.11. Nevertheless, we shall see now that, when rearranged, $\mathcal{M}^{\text {op }}$ has minimality properties for majorization, so that they are univocally determined.

Remark 3.13. Let $\gamma_{1} \geq \ldots \geq \gamma_{p} \in \mathbb{R}$ and consider $\lambda=\left(\gamma_{1} \mathbb{1}_{r_{1}}, \ldots, \gamma_{p} \mathbb{1}_{r_{p}}\right)=\left(\lambda_{i}\right)_{i \in \mathbb{I}_{r}} \in\left(\mathbb{R}^{r}\right)^{\downarrow}$, where $r \stackrel{\text { def }}{=} \sum_{i \in \mathbb{I}_{p}} r_{i}$. Set $s_{k}=\sum_{j \in \mathbb{I}_{k}} r_{j}$, for $k \in \mathbb{I}_{p}$. Given $\beta \in\left(\mathbb{R}^{r}\right)^{\downarrow}$ such that $\operatorname{tr}(\lambda)=\operatorname{tr}(\beta)$ then

$$
\lambda \prec \beta \Longleftrightarrow \sum_{i \in \mathbb{I}_{k}} \gamma_{i} r_{i} \leq \sum_{j \in \mathbb{I}_{s_{k}}} \beta_{j}, \quad \text { for } \quad k \in \mathbb{I}_{p-1} \text {. }
$$

Indeed, if the right conditions hold and there exists $0 \leq k \leq p-1$ with $s_{k}<t<s_{k+1}\left(s_{0}=0\right)$ and such that $\sum_{j \in \mathbb{I}_{t}} \lambda_{j}>\sum_{j \in \mathbb{I}_{t}} \beta_{j}$, it is easy to see that

$$
\sum_{j=s_{k}+1}^{t} \beta_{j}<\sum_{j=s_{k}+1}^{t} \lambda_{j}=\left(t-s_{k}\right) \gamma_{k+1} \Longrightarrow \beta_{t}<\gamma_{k+1} \Longrightarrow \sum_{j \in \mathbb{I}_{s_{k+1}}} \beta_{j}<\sum_{i \in \mathbb{I}_{k+1}} \gamma_{i} r_{i},
$$

which contradicts our assumption (24). Therefore $\lambda \prec \beta$.

We can now state our second main result.

Theorem 3.14. Let $\mathbf{d}=\left(d_{j}\right)_{j \in \mathbb{I}_{m}} \in\left(\mathbb{N}^{m}\right)^{\downarrow}$ and $\alpha=\left(\alpha_{i}\right)_{i \in \mathbb{I}_{n}} \in\left(\mathbb{R}_{\geq 0}^{n}\right)^{\downarrow}$ be such that $d_{1} \leq n$.

1. If we let $\mathcal{M}^{\mathrm{op}}$ be as in Definition 3.12 then, $\left(\alpha, \mathcal{M}^{\mathrm{op}}\right)$ is admissible. In particular, there exists $\Phi^{\mathrm{op}}=\left(\mathcal{F}_{j}^{\mathrm{op}}\right)_{j \in \mathbb{I}_{m}} \in \mathcal{D}(\alpha, \mathbf{d})$ such that $\mathcal{M}^{\mathrm{op}}=\mathcal{M}_{\Phi^{\mathrm{op}}}$.

2. If $\Phi=\left(\mathcal{F}_{j}\right)_{j \in \mathbb{I}_{m}} \in \mathcal{D}(\alpha, \mathbf{d})$, then

$$
\Lambda_{\Phi \text { op }} \prec \Lambda_{\Phi},
$$

where $\Lambda_{\Phi^{\mathrm{op}}}, \Lambda_{\Phi} \in \mathbb{R}_{\geq 0}^{d}$ are as in Definition 3.7. 
Proof. 1. By Definition 3.12, each vector $\mu_{i}^{\mathrm{op}} \oplus 0_{d_{1}-d_{i}}=\mu_{1}^{\mathrm{op}} \circ\left(\mathbb{1}_{d_{i}} \oplus 0_{d_{1}-d_{i}}\right)$. Then

$$
\sigma_{\mathcal{M}^{\mathrm{op}}}=\sum_{i \in \mathbb{I}_{m}}\left(\mu_{i}^{\mathrm{op}} \oplus 0_{d_{1}-d_{i}}\right)=\mu_{1}^{\mathrm{op}} \circ \sum_{i=1}^{m}\left(\mathbb{1}_{d_{i}} \oplus 0_{d_{1}-d_{i}}\right) \stackrel{(15)}{=} \mu_{1}^{\mathrm{op}} \circ h,
$$

using the vector $h=\left(h_{i}\right)_{i \in \mathbb{I}_{d_{1}}}$ defined from $\mathbf{d}$ as in Eqs. (14) and (15). Therefore, by Eq. (19) in Theorem 3.11, $\sigma_{\mathcal{M}^{\mathrm{op}}} \succ \alpha$, so the statement follows from Theorem 3.5.

2. Let $\Phi=\left(\mathcal{F}_{j}\right)_{j \in \mathbb{I}_{m}} \in \mathcal{D}(\alpha, \mathbf{d})$ be such that $\mathcal{F}_{j}=\left\{f_{i j}\right\}_{i \in \mathbb{I}_{n}}$, for $j \in \mathbb{I}_{m}$. Then

$$
\sum_{j \in \mathbb{I}_{m}}\left\|f_{i j}\right\|^{2}=\alpha_{i} \quad \text { for } \quad i \in \mathbb{I}_{n} .
$$

On the other hand, if we denote $\lambda_{i j}=\lambda_{i}\left(S_{\mathcal{F}_{j}}\right)$ for $j \in \mathbb{I}_{m}$ and $i \in \mathbb{I}_{d_{j}}$, we also have that

$$
\left(\left\|f_{i j}\right\|^{2}\right)_{i \in \mathbb{I}_{n}} \prec \lambda\left(S_{\mathcal{F}_{j}}\right)=\left(\lambda_{i j}\right)_{i \in \mathbb{I}_{d_{j}}} \quad \text { for } \quad j \in \mathbb{I}_{m}
$$

Hence, we conclude that

$$
\sum_{i \in \mathbb{I}_{s}}\left\|f_{i j}\right\|^{2} \leq \sum_{i=1}^{\min \left\{s, d_{j}\right\}} \lambda_{i j} \quad \text { for } \quad s \in \mathbb{I}_{n} \quad \text { and } \quad j \in \mathbb{I}_{m} .
$$

Let $\Phi^{\mathrm{op}} \in \mathcal{D}(\alpha, \mathbf{d})$ be as in item 1. We also consider $p \in \mathbb{N}, g_{0}=0<g_{1}<\ldots<g_{p}=d_{1}$ and $\gamma_{1}>\ldots>\gamma_{p}>0$ as in Theorem 3.11. Let $r_{1}, \ldots, r_{p} \in \mathbb{N}$ such that $\Lambda_{\Phi^{\mathrm{op}}}^{\downarrow}=\left(\gamma_{\ell} \mathbb{1}_{r_{\ell}}\right)_{\ell \in \mathbb{I}_{p}}$ as in Eq. (16). By Remark 3.13, in order to prove that $\Lambda_{\Phi \text { op }} \prec \Lambda_{\Phi}$ it is sufficient to check that

$$
\sum_{\ell \in \mathbb{I}_{q}} r_{\ell} \gamma_{\ell} \leq \sum_{i \in \mathbb{I}_{s_{q}}}\left(\Lambda_{\Phi}\right)_{i}^{\downarrow} \quad, \quad \text { where } \quad s_{q}=\sum_{\ell \in \mathbb{I}_{q}} r_{\ell} \quad, \quad \text { for every } \quad q \in \mathbb{I}_{p-1}
$$

because $\operatorname{tr} \Lambda_{\Phi^{\mathrm{op}}}=\operatorname{tr} \alpha=\operatorname{tr} \Lambda_{\Phi}$. Fix $q \in \mathbb{I}_{p-1}$ and consider the set

$$
S_{q}=\left\{(i, j): 1 \leq i \leq \min \left\{g_{q}, d_{j}\right\}, j \in \mathbb{I}_{m}\right\} .
$$

It is easy to see, using Eq. (15) (or looking at the rows and columns of the Figure 1), that

$$
\# S_{q}=\sum_{j \in \mathbb{I}_{m}} \min \left\{g_{q}, d_{j}\right\} \stackrel{(15)}{=} \sum_{i \in \mathbb{I}_{g_{q}}} h_{i}=\sum_{\ell \in \mathbb{I}_{q}}\left(\sum_{i=g_{\ell-1}+1}^{g_{\ell}} h_{i}\right) \stackrel{(16)}{=} \sum_{\ell \in \mathbb{I}_{q}} r_{\ell}=s_{q} .
$$

Therefore we can show Eq. (27) as follows: For every $q \in \mathbb{I}_{p-1}$,

$$
\begin{aligned}
\sum_{(i, j) \in S_{q}} \lambda_{i j} & =\sum_{j \in \mathbb{I}_{m}} \sum_{i=1}^{\min \left\{g_{q}, d_{j}\right\}} \lambda_{i j} \stackrel{(26)}{\geq} \sum_{j \in \mathbb{I}_{m}} \sum_{i \in \mathbb{I}_{g_{q}}}\left\|f_{i j}\right\|^{2} \stackrel{(25)}{=} \sum_{i \in \mathbb{I}_{g_{q}}} \alpha_{i} \\
& =\sum_{\ell \in \mathbb{I}_{q}}\left(\sum_{i=g_{\ell-1}+1}^{g_{\ell}} \alpha_{i}\right) \stackrel{(18)}{=} \sum_{\ell \in \mathbb{I}_{q}}\left(\sum_{i=g_{\ell-1}+1}^{g_{\ell}} \gamma_{\ell} h_{i}\right) \stackrel{(16)}{=} \sum_{\ell \in \mathbb{I}_{q}} r_{\ell} \gamma_{\ell} .
\end{aligned}
$$

Since $\sum_{i \in \mathbb{I}_{s q}}\left(\Lambda_{\Phi}\right)_{i}^{\downarrow} \stackrel{(28)}{\geq} \sum_{(i, j) \in S_{q}} \lambda_{i j}$, then Eq. (27) follows, and $\Lambda_{\Phi^{\mathrm{op}}} \prec \Lambda_{\Phi}$.

Theorem 3.14 together with the argument in Remark 3.8 allow us to obtain our third main result.

Theorem 3.15. Let $\mathbf{d}=\left(d_{j}\right)_{j \in \mathbb{I}_{m}} \in\left(\mathbb{N}^{m}\right)^{\downarrow}$ and $\alpha=\left(\alpha_{i}\right)_{i \in \mathbb{I}_{n}} \in\left(\mathbb{R}_{\geq 0}^{n}\right)^{\downarrow}$ be such that $d_{1} \leq n$. 
1. Let $\Phi^{\mathrm{op}}=\left(\mathcal{F}_{j}^{\mathrm{op}}\right)_{j \in \mathbb{I}_{m}} \in \mathcal{D}(\alpha, \mathbf{d})$ be as in Theorem 3.14. If $\varphi \in \operatorname{Conv}\left(\mathbb{R}_{\geq 0}\right)$ then we have that

$$
P_{\varphi}\left(\Phi^{\mathrm{op}}\right) \leq \mathrm{P}_{\varphi}(\Phi) \quad \text { for every } \quad \Phi=\left(\mathcal{F}_{j}\right)_{j \in \mathbb{I}_{m}} \in \mathcal{D}(\alpha, \mathbf{d}) .
$$

2. Moreover, if $\Phi=\left(\mathcal{F}_{j}\right)_{j \in \mathbb{I}_{m}} \in \mathcal{D}(\alpha, \mathbf{d})$ is such that there exists $\varphi \in \operatorname{Conv}_{\mathbf{s}}\left(\mathbb{R}_{\geq 0}\right)$ for which $\Phi$ is a global minimum for $\mathrm{P}_{\varphi}$ (i.e., if equality holds in Eq. (29)), then

$$
\Lambda_{\Phi}=\Lambda_{\Phi \mathrm{op}} \quad \text { and } \quad \mathcal{M}_{\Phi}=\mathcal{M}^{\mathrm{op}}, \quad \text { so that } \quad \lambda\left(S_{\mathcal{F}_{j}}\right)=\mu_{j}^{\mathrm{op}} \in\left(\mathbb{R}_{>0}^{d_{j}}\right)^{\downarrow}
$$

and, in particular, $\mathcal{F}_{j}$ is a frame for $\mathbb{C}^{d_{j}}$ for every $j \in \mathbb{I}_{m}$.

Proof. Let $\Phi=\left(\mathcal{F}_{j}\right)_{j \in \mathbb{I}_{m}} \in \mathcal{D}(\alpha, \mathbf{d})$, then by Theorem 3.14 we know that $\Lambda_{\Phi \text { op }} \prec \Lambda_{\Phi}$. Therefore, by Remark 3.8 we get that for every $\varphi \in \operatorname{Conv}\left(\mathbb{R}_{\geq 0}\right)$,

$$
\mathrm{P}_{\varphi}\left(\Phi^{\mathrm{op}}\right)=\sum_{j \in \mathbb{I}_{m}} \mathrm{P}_{\varphi}\left(\mathcal{F}_{j}^{\mathrm{op}}\right)=\operatorname{tr}\left(\varphi\left(\Lambda_{\Phi \mathrm{op}}\right)\right) \leq \operatorname{tr}\left(\varphi\left(\Lambda_{\Phi}\right)\right)=\sum_{j \in \mathbb{I}_{m}} \mathrm{P}_{\varphi}\left(\mathcal{F}_{j}\right)=\mathrm{P}_{\varphi}(\Phi) .
$$

If $\varphi \in \operatorname{Conv}_{\mathbf{s}}\left(\mathbb{R}_{\geq 0}\right)$ and $\Phi=\left(\mathcal{F}_{j}\right)_{j \in \mathbb{I}_{m}} \in \mathcal{D}(\alpha, \mathbf{d})$ is such that equality holds in Eq. (29), let

$$
\mathcal{C}=\left\{\Lambda_{\Psi}: \Psi=\left(\mathcal{G}_{j}\right)_{j \in \mathbb{I}_{m}} \in \mathcal{D}(\alpha, \mathbf{d})\right\} \subseteq \mathbb{R}_{\geq 0}^{d} .
$$

By Lemma 3.4 it follows that $\mathcal{C}$ is a convex set. We finally introduce $F_{\varphi}: \mathcal{C} \rightarrow \mathbb{R}_{\geq 0}, F_{\varphi}(\Lambda)=$ $\operatorname{tr}(\varphi(\Lambda))$ for $\Lambda \in \mathcal{C}$. Since $\varphi$ is strictly convex we immediately see that $F$ - which is defined on the convex set $\mathcal{C}$ - is strictly convex as well. Hence, there exists a unique $\Lambda_{\varphi} \in \mathcal{C}$ such that

$$
F\left(\Lambda_{\varphi}\right)=\min \{F(\Lambda): \Lambda \in \mathcal{C}\} .
$$

Notice that by hypothesis, we have that $F\left(\Lambda_{\Phi}\right)=F\left(\Lambda_{\Phi \text { op }}\right)=\min \{F(\Lambda): \Lambda \in \mathcal{C}\}$ so then

$$
\left(\lambda\left(S_{\mathcal{F}_{j}}\right)\right)_{j \in \mathbb{I}_{m}}=\Lambda_{\Phi}=\Lambda_{\varphi}=\Lambda_{\Phi \mathrm{op}}=\left(\lambda\left(S_{\mathcal{F}_{j}^{\mathrm{op}}}\right)\right)_{j \in \mathbb{I}_{m}} .
$$

Remark 3.16. As a consequence of Eq.'s (29) and (30), the sequence $\mathcal{M}^{\text {op }}$ of Definition 3.12 and the indexes $\left(g_{i}\right)_{i \in \mathbb{I}_{p}}$ of Theorem 3.11 are univocally determined.

On the other hand, with the notation of Theorem 3.15, if we assume that $m=1$, then the previous theorem recovers the main results from $[9,24,25,26]$. In this case, the optimal spectra $\mu^{\text {op }}$ is obtained in terms of the water-filling construction. Hence, our results can be considered as a multivariated extension of the water-filling construction (see [26]).

\section{Final comments and examples}

\subsection{On the weight partitions}

By Theorem 3.15 and Remark 3.16, the spectral structure of all $(\alpha, \mathbf{d})$-designs that minimize a strictly convex potential on $\mathcal{D}(\alpha, \mathbf{d})$ is unique. It is natural to wonder whether the $(\alpha, m)$-weight partitions corresponding to such minimizers also coincide. It turns out that this is not the case, as we shall see in the following example:

Let $\alpha=\mathbb{1}_{6} \in\left(\mathbb{R}_{>0}^{6}\right)^{\downarrow}, m=2$ and let $\mathbf{d}=(4,2) \in \mathbb{N}^{2}$. In this case $\mathcal{M}^{\text {op }}$ is given by

$$
\mu_{1}^{\mathrm{op}}=\mathbb{1}_{4} \quad \text { and } \quad \mu_{2}^{\mathrm{op}}=\mathbb{1}_{2},
$$

since $\alpha=\mathbb{1}_{6} \prec(2,2,1,1)=\sigma_{\mathcal{M}^{\text {op }}}$ (so that $\mathcal{M}^{\text {op }}$ is $(\alpha, \mathbf{d}$ )-admissible), and because the associated vector $\Lambda^{\mathrm{op}}=\left(\mu_{1}^{\mathrm{op}}, \mu_{2}^{\mathrm{op}}\right)=\mathbb{1}_{6}$ is minimal for majorization. Let

$$
A_{1}=\left(\frac{4}{6} \mathbb{1}_{6}, \frac{2}{6} \mathbb{1}_{6}\right) \in P_{\alpha, 2} \subseteq \mathcal{M}_{6,2}(\mathbb{C}) \quad \text { and } \quad A_{2}=\left(c_{1}\left(A_{2}\right), c_{2}\left(A_{2}\right)\right) \in P_{\alpha, 2}
$$


where $c_{1}\left(A_{2}\right)=\left(\mathbb{1}_{4}, 0_{2}\right)$ and $c_{2}\left(A_{2}\right)=\left(0_{4}, \mathbb{1}_{2}\right)$. It is easy to see that both matrices satisfy Eq. (8) in Lemma 3.4. Thus, we can construct $\Phi^{1}=\left(\mathcal{F}_{1}^{1}, \mathcal{F}_{2}^{1}\right) \in \mathcal{D}(\alpha, \mathbf{d})$ with weight partition $A_{1}$, in such a way that $\mathcal{F}_{1}^{1}$ is a Parseval frame for $\mathbb{C}^{4}$ and $\mathcal{F}_{2}^{1}$ is a Parseval frame for $\mathbb{C}^{2}$ (both of 6 vectors). On the other hand, if we let $\left\{e_{\ell}^{(k)}\right\}_{\ell \in \mathbb{I}_{k}}$ denote the canonical basis of $\mathbb{C}^{k}$ for $k \in \mathbb{N}$ and let $\Phi^{2}=\left(\mathcal{F}_{1}^{2}, \mathcal{F}_{2}^{2}\right) \in \mathcal{D}(\alpha, \mathbf{d})$ with

$$
\mathcal{F}_{1}^{2}=\left\{e_{1}^{(4)}, \ldots, e_{4}^{(4)}, 0,0\right\} \in\left(\mathbb{C}^{4}\right)^{6} \quad \text { and } \quad \mathcal{F}_{2}^{2}=\left\{0,0,0,0, e_{1}^{(2)}, e_{2}^{(2)}\right\} \in\left(\mathbb{C}^{2}\right)^{6},
$$

then $\Phi^{2}$ has weight partition $A_{2}$. Clearly $\mathcal{M}_{\Phi_{1}}=\mathcal{M}_{\Phi_{2}}=\mathcal{M}^{\mathrm{op}}$. That is, $A_{1}$ and $A_{2}$ are both associated to optimal $(\alpha, \mathbf{d})$-designs. Thus, weight partitions inducing optimal $(\alpha, \mathbf{d})$-designs are not unique. Note that the $(\alpha, \mathbf{d})$-designs $\Phi^{1}$ and $\Phi^{2}$ are qualitatively different.

Remark 4.1. The fact that there are many $(\alpha, m)$-partition matrices $A \in P_{\alpha, m}$ that are associated to minimizers, as in the previous example, shows that the construction of optimal $(\alpha, \mathbf{d})$-designs can not be reduced to a convex optimization problem in the space $P_{\alpha, m}$ of $(\alpha, m)$-weight partitions.

We remark that in a previous version of this paper we constructed an algorithm which produced a particular matrix $A^{\mathrm{op}} \in P_{\alpha, m}$ associated to a minimizer. That is, once $A^{\mathrm{op}}$ was constructed we considered the so-called water-filling of the columns $c_{j}\left(A^{\mathrm{op}}\right)$ in dimension $d_{j}$ (see [27]), which lead to the optimal spectra $\mu_{j}^{\mathrm{op}}$, for $j \in \mathbb{I}_{m}$.

Our new strategy, based on Theorem 3.5, allow us to compute directly the optimal spectra $\mu_{j}^{\text {op }}$ for $j \in \mathbb{I}_{m}$ (and to show the existence of $(\alpha, \mathbf{d})$-designs with these spectra). Once the optimal spectra are computed then, using Remark 3.6, we can compute several associated weight partitions that in turn allow us to compute optimal $(\alpha, \mathbf{d})$-designs in an effective way (see Section 4.3 below). This new approach has decreased considerably the length of the exposition of our results herein.

\subsection{A compact description of the problem}

There is a reformulation of the problems of this paper in a more concise model. Let $\alpha$ and $\mathbf{d}$ be as in Definition 3.1. Set $d=\operatorname{tr} \mathbf{d}$ and assume that $\mathcal{H}=\mathbb{C}^{d}=\bigoplus_{j \in \mathbb{I}_{m}} \mathcal{H}_{j}$ for some subspaces with $\operatorname{dim} \mathcal{H}_{j}=d_{j}$, for $j \in \mathbb{I}_{m}$. Let us denote by $P_{j}: \mathcal{H} \rightarrow \mathcal{H}_{j} \subseteq \mathcal{H}$ the corresponding projections.

Notice that a sequence $\mathcal{G}=\left\{g_{i}\right\}_{i \in \mathbb{I}_{n}} \in \mathcal{B}_{\alpha, d} \subseteq \mathcal{H}^{n} \Longleftrightarrow$ the sequence $\Phi=\left(\mathcal{F}_{j}\right)_{j \in \mathbb{I}_{m}}$ determined by $\mathcal{F}_{j}=P_{j}(\mathcal{G})$ (i.e. $\left.f_{i j}=P_{j}\left(g_{i}\right) \in \mathcal{H}_{j} \cong \mathbb{C}^{d_{j}}, i \in \mathbb{I}_{n}\right)$ for $j \in \mathbb{I}_{m}$, satisfies that $\Phi \in \mathcal{D}(\alpha, \mathbf{d})$.

Consider the pinching map $\mathcal{C}_{\mathbf{d}}: \mathcal{M}_{d}(\mathbb{C}) \rightarrow \mathcal{M}_{d}(\mathbb{C})$ given by $\mathcal{C}_{\mathbf{d}}(A)=\sum_{j \in \mathbb{I}_{m}} P_{j} A P_{j}$, for every $A \in \mathcal{M}_{d}(\mathbb{C})$. Then, for each $\varphi \in \operatorname{Conv}\left(\mathbb{R}_{\geq 0}\right)$ we can define a d-pinched potential

$$
\mathrm{P}_{\varphi, \mathbf{d}}(\mathcal{G}) \stackrel{\text { def }}{=} \operatorname{tr} \varphi\left(\mathcal{C}_{\mathbf{d}}\left(S_{\mathcal{G}}\right)\right) \quad \text { for every } \quad \mathcal{G} \in \mathcal{H}^{n}
$$

which describes simultaneously the behavior of the projections of $\mathcal{G}$ to each subspace $\mathcal{H}_{j}$. Actually, with the previous notations,

$$
\mathrm{P}_{\varphi, \mathbf{d}}(\mathcal{G})=\sum_{j \in \mathbb{I}_{m}} \operatorname{tr} \varphi\left(P_{j} S_{\mathcal{G}} P_{j}\right)=\sum_{j \in \mathbb{I}_{m}} \mathrm{P}_{\varphi}\left(\mathcal{F}_{j}\right)=\mathrm{P}_{\varphi}(\Phi)
$$

Therefore the problem of finding optimal $(\alpha, \mathbf{d})$-designs (and studying their properties) translates to the study of sequences $\mathcal{G} \in \mathcal{B}_{\alpha, d}$ which minimize the d-pinched potentials $\mathrm{P}_{\varphi, \mathbf{d}}$.

We point out that for $\varphi \in \operatorname{Conv}\left(\mathbb{R}_{\geq 0}\right)$ and $\mathcal{G} \in \mathcal{H}^{n}$

$$
\mathrm{P}_{\varphi, \mathbf{d}}(\mathcal{G}) \leq \operatorname{tr} \varphi\left(S_{\mathcal{G}}\right) \quad \text { but } \quad \mathrm{P}_{\varphi, \mathbf{d}}(\mathcal{G}) \neq \operatorname{tr} \varphi\left(S_{\mathcal{G}}\right) \quad \text { in general }
$$

(see Definition 2.6). Therefore, previous results related with the structure of minimizers of convex potentials in $\mathcal{B}_{\alpha, d}$ (e.g. [24]) do not apply to the d-pinched potential and we require a new approach to study this problem, as shown in Example 4.3. In the paper we use the more complicated notation of sequences because it has proved to be more useful for all the computations detailed before. 


\subsection{Examples}

Theorems 3.5, 3.14 and 3.15, combined with Remark 3.6 allow us to describe a finite step algorithmic process for the effective construction of optimal designs $\Phi^{\mathrm{op}} \in \mathcal{D}(\alpha, \mathbf{d})$ from the initial data $(\alpha, \mathbf{d})$.

A possible scheme for the algorithmic procedure would be as follows:

Algorithm 4.2. IMPUT DATA: $(\alpha, \mathbf{d})$.

STEP 1. Along with the computation of the vector $h$, by means of an iterative process, we compute the value of $s^{*}$ defined in Theorem 3.11. If $s^{*}=1$, we set $p=1, g_{1}=1$ and $\gamma_{1}=Q_{1}$. In this case, $\gamma_{1} \mathbb{1}_{d_{1}} \circ h \succ \alpha$ and go to STEP 3 . Otherwise, set $j=1$ and continue with

STEP 2. By computing the maximum among the means $P_{j, k}, j \leq k \leq s^{*}-1$ we compute $g_{j}$ and $\gamma_{j}$ as it is indicated in Theorem 3.11. If $g_{j}=s^{*}-1$, rename $j=p-1$, and $\gamma_{p}=Q_{g_{p-1}+1}$ and go to STEP 3. Otherwise, set $j=g_{j}+1$ and repeat STEP 2. This step produces the set of optimal spectra $\mathcal{M}^{\mathrm{op}}=\left\{\mu_{j}^{\mathrm{op}}\right\}_{j \in \mathbb{I}_{m}}$, such that $(\alpha, \mathcal{M})$ is admissible.

STEP 3. Apply the finite step algorithm described in Remark 3.6 and obtain $\Phi^{\mathrm{op}}=\left(\mathcal{F}_{j}^{\mathrm{op}}\right)_{j \in \mathbb{I}_{m}}$ (as described in Theorem 3.14) as output.

The following examples were obtained via an implementation of Algorithm 4.2 using MATLAB.

Example 4.3. Consider the family of weights given by $\alpha=\{9,8,7,5,4,2.5,2,2,1.5,0.6,0.5\}$ and suppose that the dimensions to be considered are $\mathbf{d}=(7,5,3)$. In this case, the optimal spectra $\mathcal{M}^{\mathrm{op}}$ are determined, as in Definition 3.12, by

$$
\mu_{1}^{\text {op }}=(3,2.7583,2.7583,2.7583,2.7583,2.7583,2.7583) .
$$

If $\sigma_{\mathcal{M}^{\mathrm{op}}} \in \mathbb{R}^{7}$ is defined as in Theorem 3.5, then, $\alpha \prec \sigma_{\mathcal{M}^{\mathrm{op}}}$ by Theorem 3.14. Using Remark 3.6 we construct $D \in \mathcal{D S}(11)$ such that $D\left(\sigma_{\mathcal{M}^{\circ p}} \oplus 0_{4}\right)=\alpha$. Setting $A \in \mathcal{M}_{11,3}\left(\mathbb{R}_{\geq 0}\right)$, such that $c_{j}(A)=D\left(\mu_{j}^{\mathrm{op}} \oplus 0_{11-d_{j}}\right) \in \mathbb{R}_{\geq 0}^{11}$, for $j=1,2,3$, we get (for example) the following partition of $\alpha$ :

$$
A=\left[\begin{array}{rrr}
3 & 3 & 3 \\
2.7583 & 2.7583 & 2.4833 \\
2.7583 & 2.7583 & 1.4833 \\
2.7583 & 1.8135 & 0.4282 \\
2.5267 & 1.1307 & 0.3425 \\
1.5792 & 0.7067 & 0.2141 \\
1.2634 & 0.5654 & 0.1713 \\
1.2634 & 0.5654 & 0.1713 \\
0.9475 & 0.4240 & 0.1285 \\
0.3790 & 0.1696 & 0.0514 \\
0.3158 & 0.1413 & 0.0428
\end{array}\right] \in P_{\alpha}, 3
$$

Once we have the partitions and optimal spectra, we can construct examples of frames using these data, applying known algorithms like one-sided Bendel-Mickey algorithm (see [10, 11, 14, 16]):

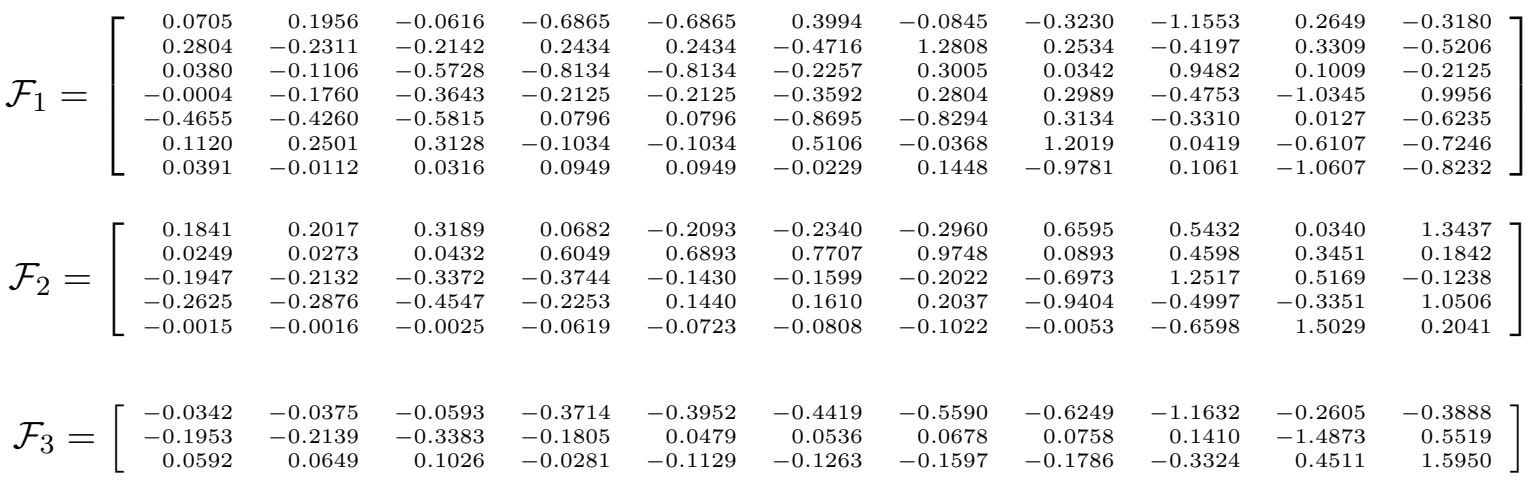


Let $\Phi^{\mathrm{op}}=\left(\mathcal{F}_{1}, \mathcal{F}_{2}, \mathcal{F}_{3}\right) \in \mathcal{D}(\alpha, \mathbf{d})$. Then, by Theorem 3.15 we have that

$$
\min \left\{\mathrm{P}_{\varphi}(\Phi): \Phi \in \mathcal{D}(\alpha, \mathbf{d})\right\}=\mathrm{P}_{\varphi}\left(\Phi^{\mathrm{op}}\right)=3 \varphi(3)+12 \varphi(2.7583) .
$$

Now, with the notation and terminology of Section 4.2, we get the following lower bound for the d-pinched potential (notice that $d=d_{1}+d_{2}+d_{3}=15$ )

$$
\min \left\{\operatorname{tr} \varphi\left(S_{\mathcal{G}}\right): \mathcal{G} \in \mathcal{B}_{\alpha, 15}\right\} \geq \min \left\{\mathrm{P}_{\varphi, \mathbf{d}}(\mathcal{G}): \mathcal{G} \in \mathcal{B}_{\alpha, 15}\right\}=3 \varphi(3)+12 \varphi(2.7583) .
$$

Indeed, since $d=15>n=11$, we have that (see [24])

$$
\min \left\{\operatorname{tr} \varphi\left(S_{\mathcal{G}}\right): \mathcal{G} \in \mathcal{B}_{\alpha, 15}\right\}=\sum_{j \in \mathbb{I}_{11}} \varphi\left(\alpha_{j}\right)+4 \varphi(0)
$$

Moreover, in case $\varphi \in \operatorname{Conv}_{\mathrm{S}}\left(\mathbb{R}_{\geq 0}\right)$ then the minimizers of the potential $\mathcal{G} \mapsto \operatorname{tr} \varphi\left(S_{\mathcal{G}}\right)$ in $\mathcal{B}_{\alpha, 15}$ are sequences $\mathcal{G}=\left\{g_{i}\right\}_{i \in \mathbb{I}_{11}} \in \mathcal{H}^{11}$ of mutually orthogonal vectors. If we further choose $\varphi(x)=x^{2}$, $x \geq 0$, then the reader can check that nor the minimal value, nor the geometric structure of minimizers of Eqs. (31) and (32) coincide.

Example 4.4. When $\alpha=\{20,19.5,10,5,4.5,3,2.4,2\}$ and $\mathbf{d}=\{5,4,4,3,2\}$, Algorithm 4.2 constructs the optimal spectra given by

$$
\begin{aligned}
& \mu_{1}^{\text {op }}=(4,3.9,3.3625,3.3625,3.3625) \\
& \mu_{2}^{\text {op }}=(4,3.9,3.3625,3.3625) \\
& \mu_{3}^{\text {op }}=(4,3.9,3.3625,3.3625) \\
& \mu_{4}^{\text {op }}=(4,3.9,3.3625) \\
& \mu_{5}^{\text {op }}=(4,3.9)
\end{aligned}
$$

where the smaller spectrum does not have the constant 3.3625. As before, using Remark 3.6, we obtain the following partition:

$$
A=\left[\begin{array}{rrrrr}
4 & 4 & 4 & 4 & 4 \\
3.9 & 3.9 & 3.9 & 3.9 & 3.9 \\
3.3625 & 2.8875 & 2.5 & 1.25 & 0 \\
1.9896 & 1.1354 & 1.25 & 0.625 & 0 \\
1.7907 & 1.0218 & 1.125 & 0.5625 & 0 \\
1.1938 & 0.6812 & 0.75 & 0.375 & 0 \\
0.955 & 0.545 & 0.6 & 0.3 & 0 \\
0.7959 & 0.4541 & 0.5 & 0.25 & 0
\end{array}\right] \in P_{\alpha, 5}
$$

Acknowledgements: We would like to thank the reviewers for several comments and suggestions that helped us to improve the contents of this manuscript.

\section{References}

[1] J. Antezana, P. Massey, M. Ruiz and D. Stojanoff, The Schur-Horn theorem for operators and frames with prescribed norms and frame operator, Illinois J. Math., 51 (2007), 537-560.

[2] M.J. Benac, P. Massey, D. Stojanoff, Convex potentials and optimal shift generated oblique duals in shift invariant spaces. J. Fourier Anal. Appl. 23 (2017), no. 2, 401-441.

[3] M.J. Benac, P. Massey, D. Stojanoff, Frames of translates with prescribed fine structure in shift invariant spaces. J. Funct. Anal. 271 (2016), no. 9, 2631-2671.

[4] J.J. Benedetto, M. Fickus, Finite normalized tight frames. Frames. Adv. Comput. Math. 18 (2003), no. 2-4, 357-385. 
[5] R. Bhatia, Matrix Analysis, Berlin-Heildelberg-New York, Springer 1997.

[6] B.G. Bodmann, V.I. Paulsen, Frames, graphs and erasures. Linear Algebra Appl. 404 (2005), 118-146.

[7] P.G. Casazza, The art of frame theory, Taiwanese J. Math. 4 (2000), no. 2, 129-201.

[8] P.G. Casazza, Custom building finite frames. In Wavelets, frames and operator theory, volume 345 of Contemp. Math., Amer. Math. Soc., Providence, RI, 2004, 61-86.

[9] P.G. Casazza, M. Fickus, J. Kovacevic, M.T. Leon, J.C. Tremain, A physical interpretation of tight frames. Harmonic analysis and applications, 51-76, Appl. Numer. Harmon. Anal., Birkhäuser Boston, MA, 2006.

[10] J. Cahill, M. Fickus, D.G. Mixon, M.J. Poteet, N. Strawn, Constructing finite frames of a given spectrum and set of lengths, Appl. Comput. Harmon. Anal. 35 (2013), 52-73.

[11] P.G. Casazza, and M.T. Leon, Existence and construction of finite frames with a given frame operator. Int. J. Pure Appl. Math. 63 (2010), no. 2, 149-157.

[12] P. G. Casazza and G. Kutyniok eds., Finite Frames: Theory and Applications. Birkhauser, 2012. xii + 483 pp.

[13] O. Christensen, An introduction to frames and Riesz bases. Applied and Numerical Harmonic Analysis. Birkhäuser Boston, 2003. xxii+440 pp.

[14] I.S. Dhillon, R.W. Heath Jr., M.A. Sustik, J.A. Tropp, Generalized finite algorithms for constructing Hermitian matrices with prescribed diagonal and spectrum, SIAM J. Matrix Anal. Appl. 27 (1) (2005) 61-71.

[15] K. Dykema, D. Freeman, K. Kornelson, D. Larson, M. Ordower, E. Weber, Ellipsoidal tight frames and projection decomposition of operators: Illinois J. Math. 48 (2004), 477-489.

[16] D. J. Feng, L. Wang and Y. Wang, Generation of finite tight frames by Householder transformations. Adv Comput Math 24 (2006), 297-309.

[17] M. Fickus, J. Marks, M. Poteet, A generalized Schur-Horn theorem and optimal frame completions. Appl. Comput. Harmon. Anal. 40 (2016), no. 3, 505-528.

[18] M. Fickus, D. G. Mixon and M. J. Poteet, Frame completions for optimally robust reconstruction, Proceedings of SPIE, 8138: 81380Q/1-8 (2011).

[19] B. Hassibi, M. Sharif, Fundamental Limits in MIMO Broadcast Channels. IEEE Journal on Selected Areas in Communications 25(7) (2007), 1333-1344.

[20] R.B. Holmes, V.I. Paulsen, Optimal frames for erasures. Linear Algebra Appl. 377 (2004), 31-51.

[21] K. A. Kornelson, D. R. Larson, Rank-one decomposition of operators and construction of frames. Wavelets, frames and operator theory, Contemp. Math., 345, Amer. Math. Soc., Providence, RI, 2004, 203-214.

[22] P. Massey, N. Rios, D. Stojanoff, Frame completions with prescribed norms: local minimizers and applications.Adv. Comput. Math., in press.

[23] P. Massey, M.A. Ruiz, Tight frame completions with prescribed norms. Sampl. Theory Signal Image Process. 7 (2008), no. $1,1-13$.

[24] P. Massey, M. Ruiz; Minimization of convex functionals over frame operators. Adv. Comput. Math. 32 (2010), no. 2, 131-153.

[25] P. Massey, M. Ruiz, D. Stojanoff, Optimal dual frames and frame completions for majorization. Appl. Comput. Harmon. Anal. 34 (2013), no. 2, 201-223.

[26] P.G. Massey, M.A. Ruiz, D. Stojanoff; Optimal frame completions. Advances in Computational Mathematics 40 (2014), 1011-1042.

[27] P. Massey, M. Ruiz, D. Stojanoff; Optimal frame completions with prescribed norms for majorization. J. Fourier Anal. Appl. 20 (2014), no. 5, 1111-1140. 